\title{
Unveiling the chemistry of interstellar $\mathrm{CH}$
} \section{Spectroscopy of the $2 \mathrm{THz} N=2 \leftarrow 1$ ground state line ${ }^{\star}$}

\author{
H. Wiesemeyer ${ }^{1}$, R. Güsten ${ }^{1}$, K.M. Menten ${ }^{1}$, C.A. Durán ${ }^{1}$, T. Csengeri ${ }^{1}$, A.M. Jacob ${ }^{1}$, R. Simon $^{2}$, \\ J. Stutzki ${ }^{2}$, and F. Wyrowski ${ }^{1}$
}

${ }^{1}$ Max-Planck-Institut für Radioastronomie, Auf dem Hügel 69, 53121 Bonn, Germany
e-mail: hwi ese@mpi fr. de
${ }^{2}$ I. Physikalisches Institut, Universität zu Köln, Zülpicher Str 77, 50937 Köln, Germany

Received 22 August 2017 / Accepted 12 December 2017

\begin{abstract}
Aims. The methylidyne radical $\mathrm{CH}$ is commonly used as a proxy for molecular hydrogen in the cold, neutral phase of the interstellar medium. The optical spectroscopy of $\mathrm{CH}$ is limited by interstellar extinction, whereas far-infrared observations provide an integral view through the Galaxy. While the $\mathrm{HF}$ ground state absorption, another $\mathrm{H}_{2}$ proxy in diffuse gas, frequently suffers from saturation, $\mathrm{CH}$ remains transparent both in spiral-arm crossings and high-mass star forming regions, turning this light hydride into a universal surrogate for $\mathrm{H}_{2}$. However, in slow shocks and in regions dissipating turbulence its abundance is expected to be enhanced by an endothermic production path, and the idea of a "canonical" $\mathrm{CH}$ abundance needs to be addressed.

Methods. The $N=2 \leftarrow 1$ ground state transition of $\mathrm{CH}$ at $\lambda 149 \mu \mathrm{m}$ has become accessible to high-resolution spectroscopy thanks to the German Receiver for Astronomy at Terahertz Frequencies (GREAT) aboard the Stratospheric Observatory for Infrared Astronomy (SOFIA). Its unsaturated absorption and the absence of emission from the star forming regions makes it an ideal candidate for the determination of column densities with a minimum of assumptions. Here we present an analysis of four sightlines towards distant Galactic star forming regions, whose hot cores emit a strong far-infrared dust continuum serving as background signal. Moreover, if combined with the sub-millimeter line of $\mathrm{CH}$ at $\lambda 560 \mu \mathrm{m}$, environments forming massive stars can be analyzed. For this we present a case study on the "proto-Trapezium" cluster W3 IRS5.

Results. While we confirm the global correlation between the column densities of $\mathrm{HF}$ and those of $\mathrm{CH}$, both in arm and interarm regions, clear signposts of an over-abundance of $\mathrm{CH}$ are observed towards lower densities. However, a significant correlation between the column densities of $\mathrm{CH}$ and HF remains. A characterization of the hot cores in the W3 IRS5 proto-cluster and its envelope demonstrates that the sub-millimeter/far-infrared lines of $\mathrm{CH}$ reliably trace not only diffuse but also dense, molecular gas.

Conclusions. In diffuse gas, at lower densities a quiescent ion-neutral chemistry alone cannot account for the observed abundance of $\mathrm{CH}$. Unlike the production of $\mathrm{HF}$, for $\mathrm{CH}^{+}$and $\mathrm{CH}$, vortices forming in turbulent, diffuse gas may be the setting for an enhanced production path. However, $\mathrm{CH}$ remains a valuable tracer for molecular gas in environments reaching from diffuse clouds to sites of high-mass star formation.
\end{abstract}

Key words. ISM: abundances - ISM: clouds - ISM: lines and bands - ISM: molecules - ISM: structure

\section{Introduction}

The methylidyne radical, $\mathrm{CH}$, was the first molecule observed in space (Dunham 1937) and identified by Swings \& Rosenfeld (1937). Most $\mathrm{CH}$ originates in the diffuse gas of the cold, neutral matter $(\mathrm{CNM})$ via the slow radiative association of $\mathrm{C}^{+}$ with $\mathrm{H}_{2}$ forming $\mathrm{CH}_{2}^{+}$, which converts to $\mathrm{CH}$ via dissociative recombination $\mathrm{CH}_{2}^{+}\left(\mathrm{e}^{-}, \mathrm{H}\right) \mathrm{CH}$ (e.g., Gerin et al. 2016). Owing to the former reaction, one expects the $\mathrm{CH}$ abundance to scale with that of $\mathrm{H}_{2}$, making $\mathrm{CH}$ a proxy for molecular hydrogen. Other reaction channels are the dissociative recombination of $\mathrm{CH}_{3}^{+}$, and the photodissociation of $\mathrm{CH}_{2}$. The usefulness of $\mathrm{CH}$ as a surrogate for $\mathrm{H}_{2}$ is confirmed by Sheffer et al. (2008), who observed the $\mathrm{CH} A-X(0-0)$ band at $430 \mathrm{~nm}$. They derive $\mathrm{CH}$ column densities that range from $\sim 10^{11}$ to $\sim 10^{13} \mathrm{~cm}^{-2}$. The reference column densities of $\mathrm{H}_{2}$ were derived from UV spectra of the Lyman $B-X$ bands. However, UV and optical studies are

\footnotetext{
* The reduced spectra (Fig. 2) are only and Table 2 is also available at the CDS via anonymous ftp to cdsarc.u-strasbg.fr (130.79.128.5) or via http://cdsarc.u-strasbg.fr/viz-bin/ qcat?]/A+A/612/A37
}

restricted to distances of up to $\sim 7 \mathrm{kpc}$, with the vast majority being much smaller (e.g., Sheffer et al. 2008), owing to Galactic extinction in the UV, and to the requirement of bright background stars in the optical. Pan et al. (2005) found that there are two populations of $\mathrm{CH}$ bearing clouds, one being associated with $\mathrm{CN}$, the other one with $\mathrm{CH}^{+}$. The abundance of $\mathrm{CH}^{+}$ and subsequently $\mathrm{CH}$ may indeed be enhanced by the molecular hydrogen abstraction reaction $\mathrm{C}^{+}\left(\mathrm{H}_{2}, \mathrm{H}\right) \mathrm{CH}^{+}$. This reaction is endothermic by $0.398 \pm 0.003 \mathrm{eV}$ or about $4620 \mathrm{~K}$ (Hierl et al. 1997, while earlier measurements yielded $\sim 4300 \mathrm{~K}$, Gerlich et al. 1987). The supply activating this reaction is thought to be provided by the dissipation of turbulence and by ion-neutral drift (Godard et al. 2009) or by slow, non-dissociative shocks permeating the CNM (Gerin et al. 2016, further references therein). Indeed, Godard et al. (2014) demonstrated that at $n_{\mathrm{H}}=50 \mathrm{~cm}^{-3}$, $80 \%$ to $90 \%$ of the $\mathrm{CH}$ column density and all of the $\mathrm{CH}^{+}$arise from bursts of dissipation, while the remaining fraction is associated with gas in the ambient medium or relaxing towards it. Only at gas densities in excess of typically $100 \mathrm{~cm}^{-3}$ does the abundance of $\mathrm{CH}$ become nearly constant (Sheffer et al. 2008; Levrier et al. 2012). As a matter of fact, at $n_{\mathrm{H}}=300 \mathrm{~cm}^{-3}$, about 
$70 \%$ of the $\mathrm{CH}$ originates in ambient conditions (Godard et al. 2009).

These results notwithstanding, $\mathrm{CH}$ is commonly used as a proxy for $\mathrm{H}_{2}$. It shares this virtue with $\mathrm{HF}$, which was first detected in the ISM by Neufeld et al. (1997). Because HF is the only neutral light hydride that exothermically forms in a triatomic reaction with $\mathrm{H}_{2}$ (Neufeld et al. 2005; Zhu et al. 2002), it is considered the more pristine $\mathrm{H}_{2}$ tracer. However, unlike for $\mathrm{CH}$, its ground-state absorption often saturates, not only towards the dense molecular gas associated with star-forming regions. The same holds for the ground-state absorption of $\mathrm{OH}$ at $2.5 \mathrm{THz}$ which can be considered a secondary $\mathrm{H}_{2}$ tracer (e.g., Wiesemeyer et al. 2016). This leaves $\mathrm{CH}$ as an important proxy at high column densities. In the more diffuse gas, below $A_{\mathrm{V}}=1$, all three species are more reliable $\mathrm{H}_{2}$ tracers than $\mathrm{CO}$, which suffers from insufficient self-shielding (Gerin et al. 2016, further references therein).

These findings leave their fingerprint in the measured abundances and their mutual correlations. Thanks to the operation of spaceborne and airborne far-infrared instrumentation with adequate spectral resolution (HIFI and GREAT ${ }^{1}$ aboard the Herschel Space Observatory ${ }^{2}$ and SOFIA, respectively),

such studies can now be conducted Galaxy-wide, unlike UV and optical observations. The spectroscopy of ground-state lines of the aforementioned light hydrides, observed in absorption along sightlines towards the hot, far-infrared bright dust associated with sites of high-mass star formation (for many of which distances have been determined from maser parallaxes, e.g., Reid et al. 2014), provides a clear-cut setting to determine the column density profiles of spiral-arm crossings with a minimum of assumptions: as an indication, only $\sim 100 \mathrm{ppm}$ of the total population in diffuse clouds resides in excited states (for details we refer to Appendix D, in particular Table D.1). Historically, besides the aforementioned UV/optical studies, $\mathrm{CH}$ column densities were determined using the radio transitions between the hyperfine-split ground-state levels (e.g., Liszt \& Lucas 2002). Although the involved level populations are affected by nonLTE level occupations, the error in the derived column densities remains moderate if the transition is optically thin and inverted, and if the uncertain excitation temperature is expected to obey the condition $\left|T_{\mathrm{ex}}\right| \gg T_{\mathrm{bg}}$ (where $T_{\mathrm{bg}}$ is the temperature of the continuum background against which the radio spectrum is observed, and assuming that beam filling factors are sufficiently well known to ensure that this condition holds). The non-LTE effect is briefly discussed in Appendix D.

The installation of the upGREAT array and its extended tuning range (Risacher et al. 2016) made the transition of $\mathrm{CH}$ molecules from the $N=1, J=1 / 2$ ground state into the $N=2$, $J=3 / 2$ state accessible to observations (the energy level diagram of $\mathrm{CH}$ is shown in Fig. 1). This transition comprises two triplets of hyperfine structure lines with wavelengths near $149 \mu \mathrm{m}$, two of which were first detected with very low spectral resolution with the Kuiper Airborne Observatory (Stacey et al. 1987). With adequate spectral resolution to distinguish the features originating in different spiral arms, this line lends itself particularly well to absorption studies of diffuse and translucent clouds because it remains in absorption even in the background

\footnotetext{
1 GREAT is a development by the MPI für Radioastronomie and the KOSMA/ Universität zu Köln, in cooperation with the MPI für Sonnensystemforschung and the DLR Institut für Planetenforschung.

2 Herschel is an ESA space observatory with science instruments provided by European-led Principal Investigator consortia and with important participation from NASA.
}

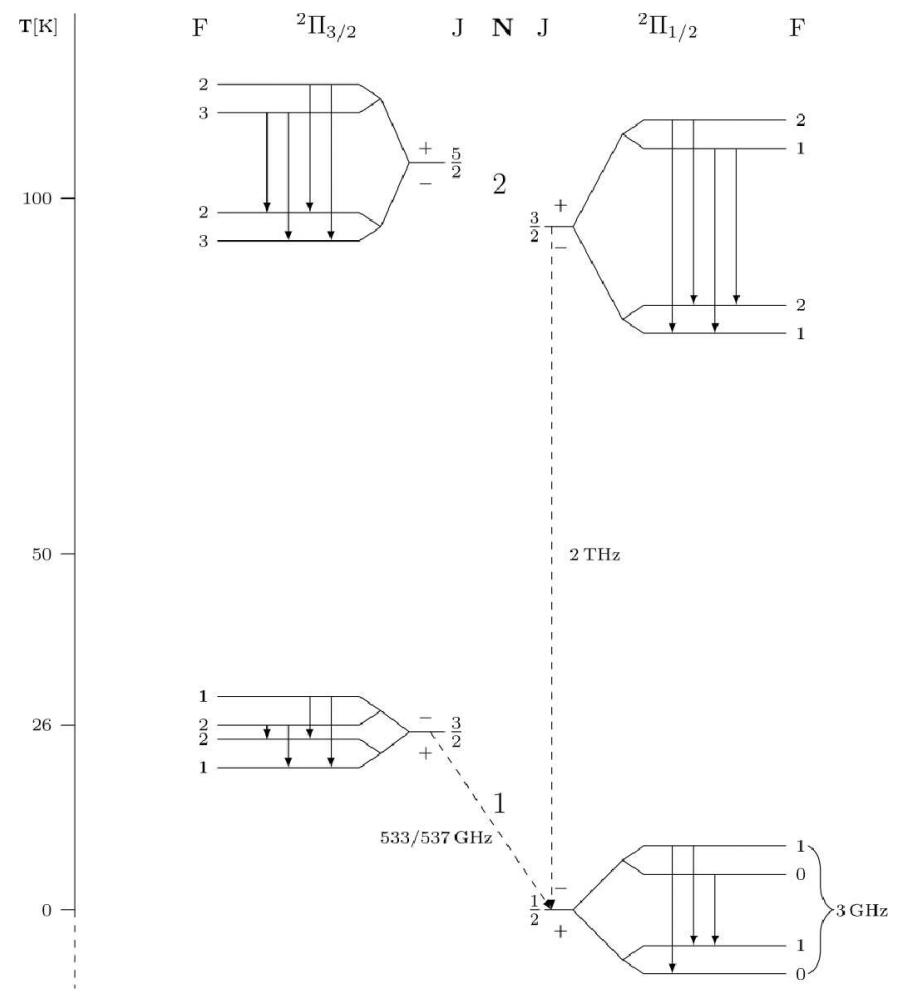

Fig. 1. Lowest rotational energy levels of the $\mathrm{CH}$ radical. The submillimeter/far-infrared transitions discussed here, at $533 / 537 \mathrm{GHz}$ (i.e., $\lambda 560 \mu \mathrm{m})$ and $2007 / 2010 \mathrm{GHz}(\lambda 149 \mu \mathrm{m})$ are indicated by the dashed arrows. Hyperfine splitting is not to scale.

source. The rotational $\lambda 560 \mu \mathrm{m}$ transition between the $N=1$, $J=3 / 2 \rightarrow 1 / 2$ shares its lower level with the ground-state transition studied here, and was detected with HIFI at high spectral resolution (Gerin et al. 2010). Because the $\lambda 560 \mu \mathrm{m}$ line of $\mathrm{CH}$ is easier to excite than its counterpart at $\lambda 149 \mu \mathrm{m}$ (cf. Fig. 1), in the environment of the background source it appears in emission, which, by virtue of its hyperfine splitting (cf. Table 1), blends with the sight-line features from spiral arms and interarm gas, rendering the analysis more difficult. In a similar context, the detection of the $A-X(0,0)$ band of $\mathrm{CH}$ near $4304 \AA$ in the environment of Herschel 36, the O-star primarily illuminating the hourglass nebula M8, reveals that its lower level, the excited ${ }^{2} \Pi, J=3 / 2$ fine structure state $25.6 \mathrm{~K}$ above ground, is significantly populated (Oka et al. 2013).

The aim of this work is therefore to establish the $\lambda 149 \mu \mathrm{m}$ line as a tool allowing us to obtain $\mathrm{CH}$ (and ultimately $\mathrm{H}_{2}$ ) column densities from first principles, with a minimum of a priori knowledge required. We will compare $\mathrm{CH}$ column density profiles with those of HF (Sonnentrucker et al. 2015, further references therein) and $\mathrm{OH}$ (Wiesemeyer et al. 2016), so as to assess the usefulness of $\mathrm{CH}$ as a tracer for molecular gas in diffuse clouds. We extend our analysis to the envelopes around regions forming high-mass stars, where, despite the high densities, the $\mathrm{CH}$ absorptions at $\lambda 149 \mu \mathrm{m}$ and $\lambda 560 \mu \mathrm{m}$ do not saturate. When analyzed together with the emission in the $\lambda 560 \mu \mathrm{m}$ line, $\mathrm{CH}$ remains a suitable $\mathrm{H}_{2}$ tracer in such environments.

\section{Observations and data analysis}

The observations were performed on several SOFIA flights in the observatory's cycle 4 (F298 and F301 on 2016 May 18 and 24, respectively, F346 on 2016 November 8, and F369 on 2017 February 3). The aircraft altitudes were $41.7-42.6 \mathrm{kft}$. 
Table 1. Hyperfine splitting of the $N=2 \leftarrow 1, J=3 / 2 \leftarrow 1 / 2$ transitions.

\begin{tabular}{cccccc}
\hline \hline HFC & $\begin{array}{c}\text { Frequency } \\
{[\mathrm{GHz}]}\end{array}$ & $\begin{array}{c}A_{\mathrm{E}} \\
{\left[\mathrm{s}^{-1}\right]}\end{array}$ & $\begin{array}{c}E_{\mathrm{L}} \\
{[\mathrm{K}]}\end{array}$ & $\begin{array}{c}E_{\mathrm{U}} \\
{[\mathrm{K}]}\end{array}$ & $\begin{array}{c}n_{\text {crit }} \\
{\left[\mathrm{cm}^{-3}\right]}\end{array}$ \\
\hline$F=1^{-} \leftarrow 1^{+}$ & 2006.74892 & 0.01117 & 0.00072 & 96.31011 & \\
$F=1^{-} \leftarrow 0^{+}$ & 2006.76263 & 0.02234 & 0.00000 & 96.31005 & $\sim 3 \times 10^{10}$ \\
$F=2^{-} \leftarrow 1^{+}$ & 2006.79912 & 0.03350 & 0.00072 & 96.31252 & \\
\hline$F=1^{-} \leftarrow 1^{+}$ & 2010.73859 & 0.01129 & 0.16071 & 96.66158 & \\
$F=1^{-} \leftarrow 0^{+}$ & 2010.81046 & 0.02257 & 0.15726 & 96.66157 & $\sim 2 \times 10^{10}$ \\
$F=2^{-} \leftarrow 1^{+}$ & 2010.81192 & 0.03386 & 0.16071 & 96.66510 & \\
\hline
\end{tabular}

Notes. Data are from Pickett et al. (1998). Col. (1): hyperfine component. Col. (3): Einstein coefficient. Cols. (4) and (5): lower and upper state energy. Col. (6): critical density, $A_{\mathrm{E}} / \gamma$, for $100 \mathrm{~K}$ gas temperature. The collisional rate cofficients $\gamma$ do not account for hyperfine splitting and are scaled by reduced mass (from $\mathrm{He}$ to para- $\mathrm{H}_{2}$ as collision partner, Marinakis et al. 2015).

The configuration of GREAT was single-pixel channel L1 and upGreat's arrays LFA-H and $-\mathrm{V}$ (seven pixels each). The frequency of the $N=2 \leftarrow 1, J=3 / 2 \leftarrow 1 / 2$ transition of $\mathrm{CH}$ at $2006.762 \mathrm{GHz}$ (Table 1) was tuned to the upper sideband, so as to avoid a prominent telluric ozone feature appearing in the image band of a lower sideband tuning. Typical single-sideband receiver temperatures were $1650-1750 \mathrm{~K}$, while the signal-band opacity varied from 0.2 to 0.3 . The spectra were taken in chopnod mode, with a chop amplitude of $80^{\prime \prime}$ and a frequency of $2.5 \mathrm{~Hz}$. The $\mathrm{CH}$ absorption spectrum appears in the central pixel of the LFA-H array, while its remaining six pixels, covering a hexagonal arrangement with 33" spacing (Risacher et al. 2016, this corresponds to 2.3 times the beam width (full width at half maximum, FWHM) at the observing frequency) were used to better define the atmospheric total power emission off the source. After down-conversion to an intermediate-frequency bandpass of $4 \mathrm{GHz}$, the raw data were analyzed with XFFTS spectrometers providing $283 \mathrm{kHz}$ resolution, covering the bandpass with $2^{14}$ channels (Klein et al. 2012). The spectra were calibrated with the KALIBRATE program (Guan et al. 2012), which is part of the KOSMA package also used to execute the observations. The underlying forward and main-beam efficiencies are 0.97 and 0.68 , respectively. Further processing with the CLASS software ${ }^{3}$ included spectral smoothing to $0.36 \mathrm{~km} \mathrm{~s}^{-1}$-wide velocity bins, which is a good compromise between sensitivity and resolving power for narrow absorption features, and a correction for spectral baselines by removing polynomials of up to third order. The continuum level, determined by means of a dedicated doublesideband calibration, was added back to the spectra in order to ensure a correct line-to-continuum ratio.

In contrast to techniques applied for optical/UV spectroscopy, the analysis of rotational ground state lines arising in diffuse clouds needs no excitation or extinction corrections. At their typical temperatures of $15-100 \mathrm{~K}$, one can safely assume that the $\mathrm{CH}$ radical is almost entirely in its ${ }^{2} \Pi_{1 / 2}$ ground state (cf. Appendix D), and spontaneous de-excitation can be neglected. The opacity for a velocity component $i$ and a hyperfine component $j$ therefore reads

$\tau_{\mathrm{ij}, v}=\sqrt{\frac{\ln 2}{\pi}} \frac{A_{\mathrm{E}, j} c^{3}}{4 \pi \Delta v_{\mathrm{i}} v_{\mathrm{j}}^{3}} \frac{g_{\mathrm{u}, \mathrm{j}}}{g_{\mathrm{l}, \mathrm{j}}} N w_{\mathrm{j}} \exp \left(-4 \ln 2\left(\frac{v-v_{0, i j}}{\Delta v_{i}}\right)^{2}\right)$,

where $A_{\mathrm{E}, j}$ is the Einstein coefficient and $\Delta v_{\mathrm{i}}$ the half-power width of the Gaussian profile of the velocity component centered at $v_{0, i j}$. The degeneracies of the upper and lower level of

\footnotetext{
3 Development led by IRAM, http://www.iram.fr/IRAMFR/ GILDAS/
}

hyperfine components $j$ involved in the transition are denoted $g_{\mathrm{u}, \mathrm{j}}$ and $g_{1, \mathrm{j}}$, respectively. $N$ is the total column density of $\mathrm{CH}$ in the ground state, and the factor $w_{\mathrm{j}}=g_{1, \mathrm{j}} \exp \left(-E_{\mathrm{l}, \mathrm{j}} /(k T)\right) / Q(T)$ describes the fractional population in the hyperfine-split level $j$, at an energy $E_{1, \mathrm{j}}$ above ground and for an excitation temperature $T$, with partition function $Q(T)$.

In the following analysis, we fit ensembles of Gaussian velocity components to the opacity spectra obtained from the observations, that is, each component follows the ansatz given by Eq. (1). The numerical implementation of this method is described in Wiesemeyer et al. (2016). In view of the saturated absorption in portions of the absorption spectra of $\mathrm{OH}$ and $\mathrm{HF}$, we emphasize that such an approach implies two features: entirely saturated velocity components do not arise in the analysis, because they can evidently not constrain the minimization of the merit function. On the other hand, components of which only a minor portion is affected by saturated absorption still constrain the column density across the full velocity interval (but only for the cloud entity that they represent). The propagation of the baseline noise and of the uncertainty in the line-to-continuum ratio into the determination of column densities is discussed in Appendix A. A note on the treatment of asymmetric error distributions is given in Appendix B.

\section{Results}

\subsection{Main characteristics of the observed sightlines}

The spectra of the $\lambda 149 \mu \mathrm{m}$ transition of $\mathrm{CH}$ are shown in Fig. 2, along with the deduced velocity distributions of the column density (typically $10^{13} \mathrm{~cm}^{-2}$ ). Before we proceed to their quantitative analysis, we briefly summarize the main characteristics of the observed sightlines.

The mini-starburst template $\mathrm{W} 49 \mathrm{~N}$, forming a young massive cluster (Galván-Madrid et al. 2013), is located in the Perseus spiral arm, at $11.1 \mathrm{kpc}$ distance (Zhang et al. 2013). The sightline grazes the Sagittarius spiral arm, which displays absorption at velocities from 30 to $70 \mathrm{~km} \mathrm{~s}^{-1}$, in two groups corresponding to the near and far side. The velocity interval from -10 to $+30 \mathrm{~km} \mathrm{~s}^{-1}$ represents the Perseus spiral arm. Being close to zero velocity, these absorption components may also contain gas from the local arm, a conjecture corroborated by the sightline to W51 e1/e2 discussed below. Thanks to the $\sim 80 \mathrm{~km} \mathrm{~s}^{-1}$ wide velocity range and its length, this sightline provides a good basis for abundance studies with solid statistics (e.g., Monje et al. 2011 for the HF 1-0 line, and Gerin et al. 2010 for the $\lambda 560 \mu \mathrm{m} \mathrm{CH}$ doublet), while it avoids the complexity of the central molecular zone of the Galaxy. We therefore used this sightline to 

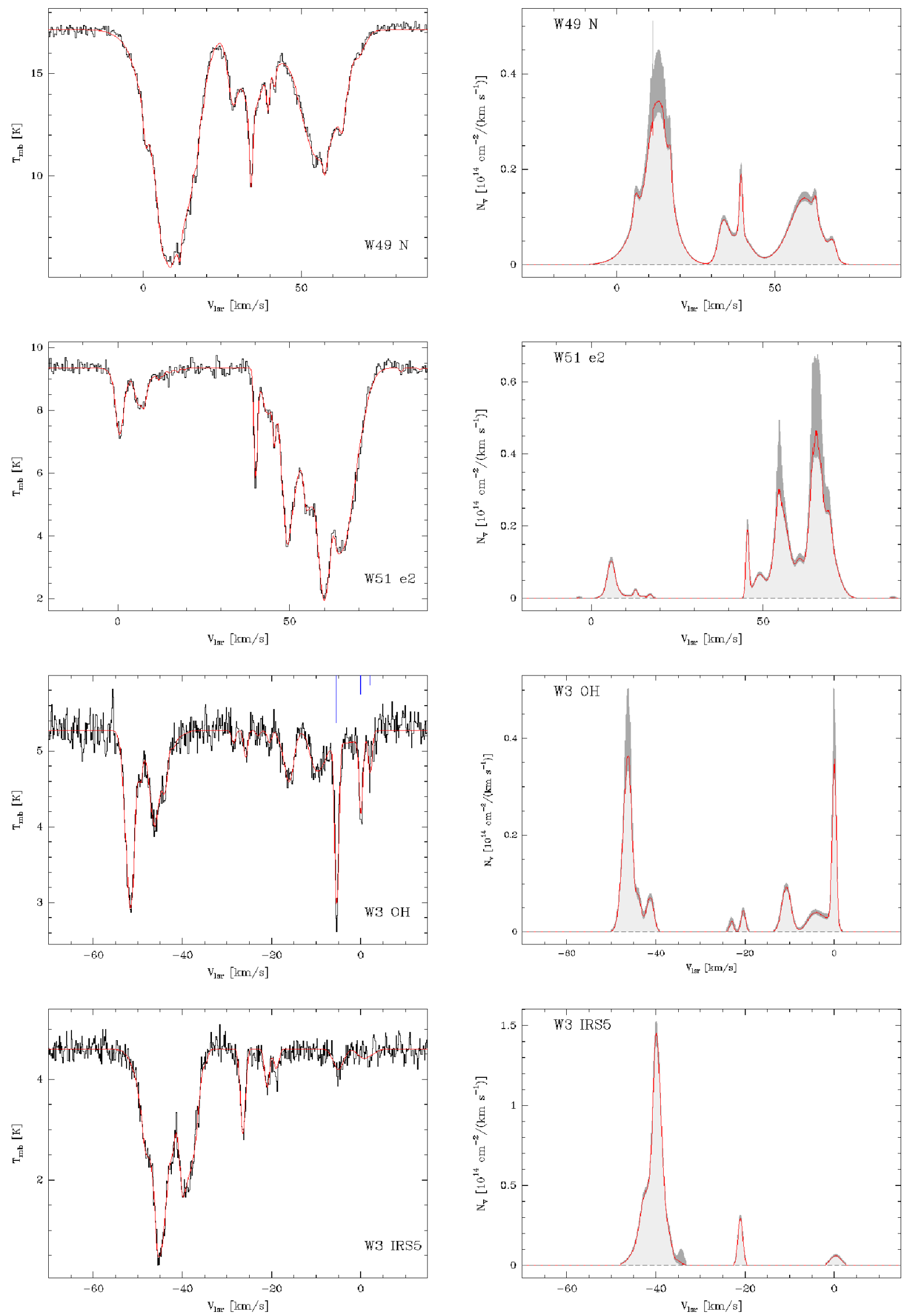

Fig. 2. $\mathrm{CH} \lambda 149 \mu \mathrm{m}$ spectra (left panel, the reference frequency for the velocity scale given by the second HFC) and deduced column density profiles (right panel) for (from top to bottom): W49 N, W51 e2, W3(OH), W3 IRS5. The blue markers in the W3(OH) spectrum show the hyperfine splitting and weights). The median column densities are indicated by the red lines. The dark-gray-shadowed profiles indicate the $\left(-\sigma_{-},+\sigma_{+}\right)$limits, that is, $84.13 \%$ confidence, assuming a $5 \%$ error in the continuum level. 
confirm that the calibration of the line-to-continuum ratios in the upGREAT and HIFI spectra is consistent (Appendix C).

The second target, W51 e1/e2 (which is part of the W51 A region), is a similar protocluster, though less massive than W49 N (e.g., Ginsburg et al. 2017). At a similar Galactic longitude, its distance of $5.4 \mathrm{kpc}$ (Sato et al. 2010) locates this complex in the near-side crossing of the Sagittarius spiral arm, where the $\mathrm{CH}$ column is distributed across a $\sim 30 \mathrm{~km} \mathrm{~s}^{-1}$-wide velocity range, separated from the gas in the $0-20 \mathrm{~km} \mathrm{~s}^{-1}$ interval by a $25 \mathrm{~km} \mathrm{~s}^{-1}$-wide gap void of $\mathrm{CH}$. The $\mathrm{CH}$ column across these low velocities must arise from gas located closer to, or located in, the solar neighborhood. It cannot arise from the Perseus arm as in the case of W49 N, given the shorter distance to $\mathrm{W} 51 \mathrm{e} 1 / \mathrm{e} 2$. The presence of $\mathrm{CH}$ in this velocity component is not surprising, since it was known to be traced by other molecules or molecular ions like $\mathrm{CH}^{+}$(Falgarone et al. 2010a), $\mathrm{OH}^{+}$and $\mathrm{OH}$ (Wiesemeyer et al. 2016), and $\mathrm{HF}$ and $\mathrm{H}_{2} \mathrm{O}$ (Sonnentrucker et al. 2015). The component consists of partially molecular, diffuse gas, since it is also present in the atomic tracers HI (Winkel et al. 2017), CII (Gerin et al. 2012, 2015) and OI (R. Güsten, 2017, priv. comm.).

The W3 complex is located in the second quadrant of the Galaxy in the Perseus arm. It consists of a classical HII region/radio continuum source associated with a prominent far infrared source. Its most prominent, high-mass star forming sub-regions are $\mathrm{W} 3(\mathrm{OH})$ and $\mathrm{W} 3$ Main (for an overview see e.g., Megeath et al. 2008); in projection they are $\sim 10 \mathrm{pc}$ apart. The archetypical ultracompact HII region W3(OH) (e.g., Qin et al. 2016) is located at a distance of $2 \mathrm{kpc}$ (Xu et al. 2006; Hachisuka et al. 2006). W3 Main contains several luminous infrared sources, out of which W3 IRS5, harboring a massive protocluster (Wang et al. 2013), is the IR-brightest. Far-infrared continuum emission from both sources has been imaged with FORCAST and SOFIA (Salgado et al. 2012; Hirsch et al. 2012). The velocity distribution of $\mathrm{CH}$ towards $\mathrm{W} 3(\mathrm{OH})$ shows two major components, of which one is attributed to the target itself and its envelope, and the other one to the local spiral arm. Towards W3 IRS5, this latter component is much less prominent. The sightlines to both targets contain a component close to $-20 \mathrm{~km} \mathrm{~s}^{-1}$, which is attributed to the near side of the Perseus arm (e.g., Vallée 2008).

\subsection{Quantitative analysis}

In the following analysis we use the HF ground-state absorption as reference for the available $\mathrm{H}_{2}$ reservoir, which we will correlate with the column densities of $\mathrm{CH}$. As already stated in the introduction, the exothermic direct association of $\mathrm{F}$ with $\mathrm{H}_{2}$ (Neufeld et al. 2005; Zhu et al. 2002) lends HF its virtue as primary $\mathrm{H}_{2}$ tracer. Here we use archival HF data from the Herschel guaranteed time key program PRISMAS ${ }^{4}$ (P.I. Maryvonne Gerin). The analysis (Fig. 3) yields $N(\mathrm{HF}) / N(\mathrm{CH})$ ratios of $0.30 \pm 0.01,0.34 \pm 0.02,0.26 \pm 0.02$ and $0.13 \pm 0.04$ towards $\mathrm{W} 49 \mathrm{~N}, \mathrm{~W} 51 \mathrm{e} 2, \mathrm{~W} 3(\mathrm{OH})$ and W3 IRS5, respectively. The corresponding correlation coefficients are $0.87,0.85,0.94$ and 0.98 .

For the evaluation of false-alarm probabilities various statistical tests exist (i.e., for the likelihood that a chance coincidence mimics a correlation between the column densities of HF and $\mathrm{CH}$ ). If we interpreted Pearson's $p$ value as false-alarm probability against our null hypothesis (the column densities of $\mathrm{CH}$ and HF being uncorrelated), our results would be significant at the $1 \%$ level. However, the usefulness of $p$ value testing has been questioned by a controversy which became known as the

\footnotetext{
4 http://astro.ens.fr/?prismas.
}

" $p$ value fallacy" (Sellke et al. 2001). We therefore provide an independent test: the non-zero column densities of $\mathrm{CH}$ are randomly permuted among the available velocity intervals (of $1 \mathrm{~km} \mathrm{~s}^{-1}$ width each), using a modified Fisher-Yates algorithm. We then count the number of false alarms (i.e., correlations detected with a $p$ threshold of 0.05 ) out of a total of 1000 Monte Carlo tests. This yields false-alarm probabilities of 4.0, $4.2,6.0$ and $7.9 \%$, respectively. If these probabilities were $p$ values, the correlations towards $\mathrm{W} 3(\mathrm{OH})$ and W3 IRS5 would merely be considered "quasi-significant". However, given that our test is more critical, it seems fair to say that all four sightlines support the conjecture that $\mathrm{HF}$ and $\mathrm{CH}$ form out of a common $\mathrm{H}_{2}$ reservoir. Assuming that the average physical conditions on these sightlines are comparable, one can also stack the data (Fig. $4)$. This yields a highly significant $p$ value $(\ll 0.01 \%)$, while the Monte Carlo analysis detects a false alarm in $4.4 \%$ of the sample, which renders the correlation significant. We performed a dedicated regression analysis, accounting for the individual errors in $N(\mathrm{HF})$ and $N(\mathrm{CH})$ using the merit function

$\chi^{2}=\sum_{i=1}^{n} \frac{\left(N_{\mathrm{HF}, \mathrm{i}}-a-b N_{\mathrm{CH}, \mathrm{i}}\right)^{2}}{\sigma_{\mathrm{HF}, \mathrm{i}}^{2}+b^{2} \sigma_{\mathrm{CH}, \mathrm{i}}^{2}}$,

(Press et al. 1992, their equation (15.3.2)) where $n$ is the number of spectral channels, and $\sigma_{\mathrm{CH}, \mathrm{i}}$ and $\sigma_{\mathrm{HF}, \mathrm{i}}$ are the root mean square (r.m.s.) errors in the individual spectral channels, as given by the distributions resulting from the Bayesian estimate. The analysis reveals that the scatter in the data is not due to sensitivity limitations or uncertainties in the line-to-continuum ratio (see Appendices A and B), but rather due to the chemical diversity on the sightlines. The consequences of this finding will be discussed in the following section.

The abundance of HF was determined by Indriolo et al. (2013) using the $2.5 \mu \mathrm{m}$ ro-vibrational transition. Sonnentrucker et al. (2015) compared the distribution of $\mathrm{HF}$ with that of $\mathrm{H}_{2} \mathrm{O}$ on twelve sightlines (further references can be found in these studies); their models predict HF abundances (with respect to $\mathrm{H}_{2}$ ) of $X(\mathrm{HF}) \sim 0.9 \times 10^{-8}$ in the low-density regime (at $A_{\mathrm{V}}=0.9$ ) to $X(\mathrm{HF})=3.3 \times 10^{-8}$ at $A_{\mathrm{V}} \sim 4$ (for further references to determinations of $X(\mathrm{HF})$ see Wiesemeyer et al. 2016). With these values, our mean $\mathrm{HF} / \mathrm{CH}$ of 0.3 translates to $X(\mathrm{CH})=0.3-1.1 \times 10^{-7}$. For comparison, the "canonical" $\mathrm{CH}-\mathrm{H}_{2}$ relationship, derived from optical and UV spectroscopy, is $N(\mathrm{CH}) / N\left(\mathrm{H}_{2}\right)=3.5_{-1.4}^{+2.1} \times 10^{-8}$ (Sheffer et al. 2008). In both studies the uncertainties reflect the chemical diversity along the sightlines, not the measurement errors. The analysis of Indriolo et al. (2013) and Sonnentrucker et al. (2015) suggests a mean abundance ratio of $N(\mathrm{HF}) / N(\mathrm{CH})=0.4$. Some of our data, at $N(\mathrm{CH}) \gtrsim 0.1 \times 10^{13} \mathrm{~cm}^{-2}$, agree with this relation (blue lines in Fig. 3). However, on the sightlines to W49 N and W51 e2, the correlation widens for $N(\mathrm{HF}) \lesssim 0.1 \times 10^{13} \mathrm{~cm}^{-2}$. We suggest that this is due to a distinctly different chemistry, leading to an over-abundance of $\mathrm{CH}$. We emphasize that the assumption of an overly low excitation temperature for the velocity intervals coinciding with the star-forming regions in the background is not the reason for this bimodal distribution: first, using a higher, more realistic excitation towards the hot cores (cf. Sect. 4.2) would simply move the data points upwards along the bisecting line without considerably changing the apparent bimodality. In the spectrum of $\mathrm{W} 49 \mathrm{~N}$, this bimodality is most striking for the data points with $N(\mathrm{CH}) \sim 0.1 \times 10^{13} \mathrm{~cm}^{-2}$, derived from sightline velocities below $v_{\mathrm{lsr}}<-1.0 \mathrm{~km} \mathrm{~s}^{-1}$. The negative velocities towards $\mathrm{W} 49 \mathrm{~N}$ are known to partially originate from expanding gas driven by an energetic outflow, evidence supported not only 


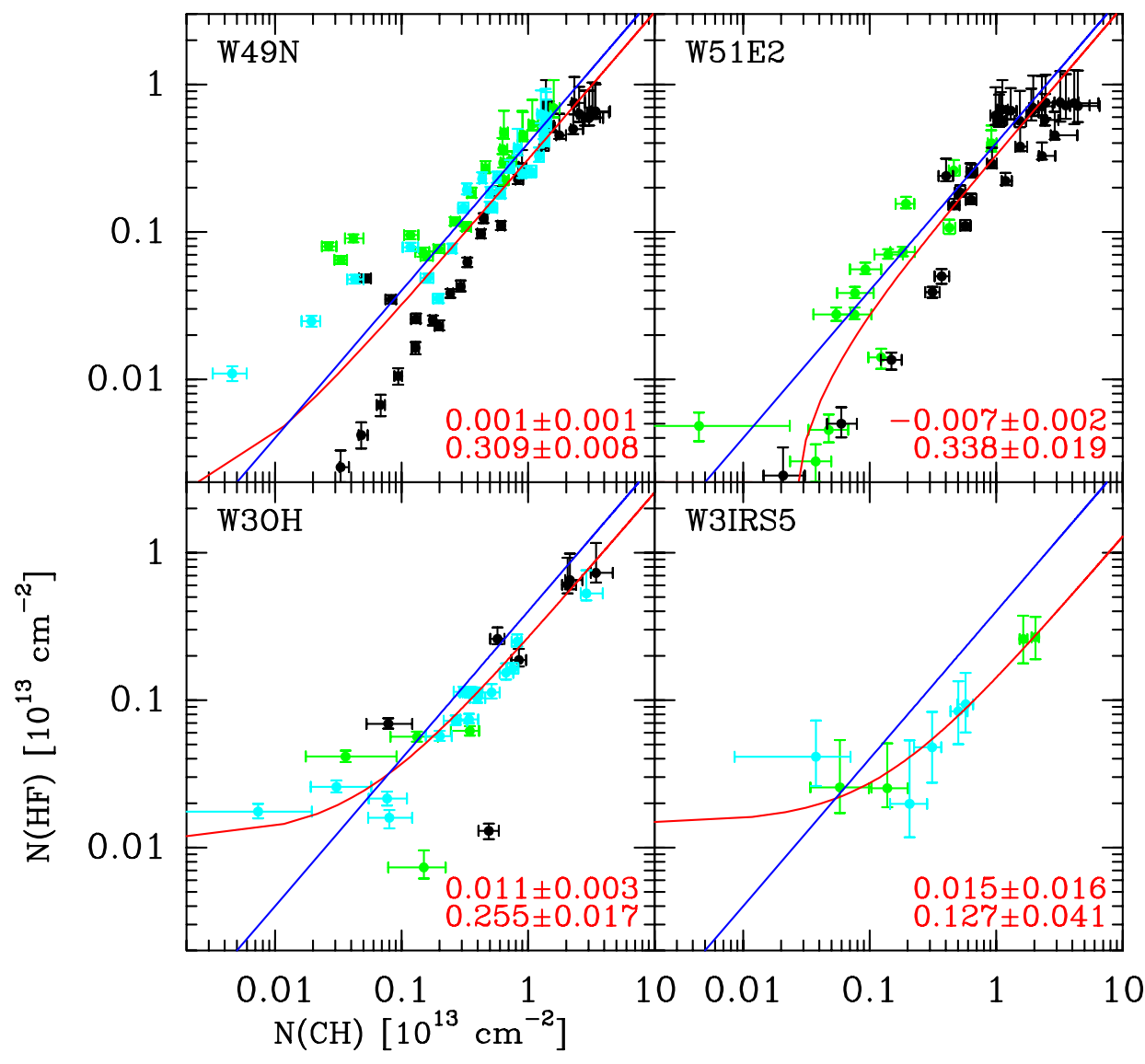

Fig. 3. Correlation between $\mathrm{HF}$ and $\mathrm{CH}$ column densities towards W49 N, W51 e2, W3 IRS5 and W3(OH) (clockwise from top left). Column densities are for $1 \mathrm{~km} \mathrm{~s}^{-1}$ wide velocity intervals (Fig. 2). Ordinate offsets and slopes from the weighted regression (parameters $a$ and $b$ in Eq. (2)) are given in the lower-right corners. The red lines show the corresponding fits, the blue lines a ratio of $\mathrm{HF} / \mathrm{CH}=0.4$ (Sheffer et al. 2008; Sonnentrucker et al. 2015). Double-logarithmic scales illustrate the bimodal distribution at low column densities towards $\mathrm{W} 49 \mathrm{~N}$ and W51 e2. Black markers indicate the absorption around, or close to, the location of the continuum source (W49 N: $v_{\mathrm{lsr}}<27 \mathrm{~km} \mathrm{~s}^{-1}$; W51 e2: $v_{\text {lsr }}>30 \mathrm{~km} \mathrm{~s}^{-1}$; W3(OH), W3 IRS5: $v_{\text {lsr }}<$ $\left.-30 \mathrm{~km} \mathrm{~s}^{-1}\right)$. Green markers indicate: for W49 N, $27 \mathrm{~km} \mathrm{~s}^{-1}<v_{\text {lsr }}<47 \mathrm{~km} \mathrm{~s}^{-1}$, for $\mathrm{W} 51 \mathrm{e} 2, v_{\text {lsr }}<30 \mathrm{~km} \mathrm{~s}^{-1}$, and for W3(OH), W3 IRS5 $-30 \mathrm{~km} \mathrm{~s}^{-1}<v_{\text {lsr }}<-15 \mathrm{~km} \mathrm{~s}^{-1}$. Remaining velocities are shown in cyan. by the wide line-wings seen in HCN (Liu et al. 2015), but also by the $\mathrm{OH}^{2} \Pi_{1 / 2}, J=3 / 2 \rightarrow 1 / 2$ emission (Menten et al., in prep.) and whose blue wing coincides with less prominent $\mathrm{p}-\mathrm{H}_{2} \mathrm{O}$ emission (Sonnentrucker et al. 2015). Notwithstanding, it is plausible that we also see unrelated (e.g., local) diffuse foreground gas at the negative velocities: first, if the absorption there was contaminated by the blue-shifted line wing related to the outflow-driven expanding gas, we would expect to also see its red-shifted counterpart (traced by the prominent emission from the excited $\mathrm{OH}$ line). This is obviously not the case; at $+28 \mathrm{~km} \mathrm{~s}^{-1}$, between the Perseus and Sagittarius spiral arms, the $\mathrm{CH}$ column density drops to almost zero. Second, towards W51 e 2 we also find three data points that exhibit a statistically significant over-abundance and that do not belong to the gas envelope in which W51 e2 is embedded. As already mentioned, they are rather located in the diffuse gas of the Sagittarius spiral arm where the assumption of a complete ground-state population is realistic, or at least inconsequential.

\section{Discussion}

\subsection{Chemistry of $\mathrm{CH}$ in diffuse gas}

The most compelling explanation for the overabundance of $\mathrm{CH}$ with respect to its canonical value is the endothermic hydrogen abstraction reaction forming $\mathrm{CH}^{+}$from $\mathrm{C}^{+}$, followed by the exothermic hydrogen abstraction reactions leading to $\mathrm{CH}_{2}^{+}$ and $\mathrm{CH}_{3}^{+}$, from where dissociative recombinations lead to the formation of $\mathrm{CH}$ and $\mathrm{CH}_{2}$, respectively. The mystery around the ubiquity of $\mathrm{CH}^{+}$, identified in the CNM by means of absorption spectroscopy in optical lines $\left({ }^{1} \Pi \rightarrow{ }^{1} \Sigma\right.$ transitions, Douglas $\&$ Herzberg 1941) and in the far-infrared $\left({ }^{1} \Sigma, J=1 \rightarrow 0\right.$ transition, Naylor et al. 2010; Falgarone et al. 2010a) was long ago discussed in the literature (e.g., Black et al. 1975; Black \& Dalgarno 1977). $\mathrm{CH}^{+}$is also found to be highly abundant in the Orion bar (Naylor et al. 2010; Parikka et al. 2017), a prototypical PDR, and in dense, strongly UV-illuminated gas in regions forming high-mass stars (Naylor et al. 2010; Falgarone et al. $2010 b)$. It is worth noting that vibrationally excited $\mathrm{H}_{2}$ makes the reaction $\mathrm{C}^{+}\left(\mathrm{H}_{2}, \mathrm{H}\right) \mathrm{CH}^{+}$exothermic (Hierl et al. 1997; Zanchet et al. 2013), and that the vibrational excitation of $\mathrm{H}_{2}$ may be chemically pumped thanks to its formation on carbonaceous dust grains (Gough et al. 1996). However, while this reaction channel plays a significant role in photo-dissociation regions (Agúndez et al. 2010; Faure et al. 2017), it cannot account for the large $\mathrm{CH}^{+}$ abundance in diffuse and translucent clouds (Agúndez et al. 2010). For these media, one mechanism suggested to overcome the endothermicity of $4620 \mathrm{~K}$ is shock chemistry (Pineau des Forets et al. 1986; Draine 1978). Another mechanism proposed for the high abundance of $\mathrm{CH}^{+}$throughout the $\mathrm{CNM}$ and compact HII regions (Naylor et al. 2010) is the dissipation of turbulence (Godard et al. 2009). In this scenario, the synthesis of $\mathrm{CH}^{+}$and, subsequently, $\mathrm{CH}$ is more efficient for lower gas densities, where more and larger vortices form in the turbulent, diffuse gas, with an increased lifetime. On the other hand, the majority of the HF column density builds up in ambient diffuse gas not exposed to dissipating turbulent cells or slow shocks (Godard et al. 2014). A closer look at the correlation between the abundances of $\mathrm{HF}$ and $\mathrm{CH}$ (Fig. 3) reveals a substantial fraction of material at $N(\mathrm{CH}) \lesssim 10^{13} \mathrm{~cm}^{-2}$ that would not be expected solely from the corresponding HF abundance. This conclusion is corroborated by the analysis of the stacked data shown in Fig. 4. On the other hand, the resulting regression coefficient $a$ does not significantly deviate from zero. Based on this data it therefore seems fair to conclude that $\mathrm{CH}$ and $\mathrm{HF}$ always coexist, but with abundance ratios varying within the limits that become 
Table 2. Synopsis of velocity-integrated column densities of $\mathrm{CH}, \mathrm{HF}$, and $\mathrm{OH}$ on the sightline to W49 N.

\begin{tabular}{lccccccc}
\hline \hline & $\begin{array}{c}v \\
{\left[\mathrm{~km} \mathrm{~s}^{-1}\right]}\end{array}$ & $N(\mathrm{CH})$ & $\begin{array}{c}N(\mathrm{HF}) \\
{\left[10^{13} \mathrm{~cm}^{-2}\right]}\end{array}$ & $N(\mathrm{OH})$ & $f_{\mathrm{H}_{2}}{ }^{d}$ & $N(\mathrm{HF}) / N(\mathrm{CH})^{e}$ & $N(\mathrm{OH}) / N(\mathrm{CH})^{e}$ \\
\hline Local gas & $(-3,+5)$ & $2.97_{-0.02}^{+0.03}$ & $0.689_{-0.007}^{+0.009}$ & $21.7_{-0.6}^{+1.9}$ & $0.32_{-0.14}^{+0.14}$ & $0.232_{-0.003}^{+0.004}$ & $7.31_{-0.24}^{+0.65}$ \\
Perseus $^{a}$ & $(+5,+20)$ & $33.2_{-0.2}^{+1.1}$ & $7.98_{-0.08}^{+0.35}$ & $61.3_{-1.9}^{+5.7}$ & $0.78_{-0.57}^{+0.16}$ & $0.240_{-0.003}^{+0.013}$ & $1.85_{-0.07}^{+0.19}$ \\
& & & & & & $3.59_{-0.09}^{+0.13}$ \\
Interarm & $(+20,+30)$ & $1.67_{-0.02}^{+0.02}$ & $0.580_{-0.004}^{+0.004}$ & $5.99_{-0.14}^{+0.21}$ & $0.31_{-0.22}^{+0.16}$ & $0.347_{-0.005}^{+0.005}$ & $3.82_{-0.08}^{+0.18}$ \\
Sgr $^{b}$ & $(+30,+45)$ & $9.91_{-0.06}^{+0.10}$ & $4.62_{-0.04}^{+0.18}$ & $37.9_{-0.8}^{+1.7}$ & $0.76_{-0.30}^{+0.20}$ & $0.466_{-0.005}^{+0.019}$ & $2.08_{-0.03}^{+0.08}$ \\
$\mathrm{Sgr}^{c}$ & $(+45,+70)$ & $18.35_{-0.09}^{+0.14}$ & $6.47_{-0.05}^{+0.20}$ & $38.1_{-0.4}^{+1.5}$ & $0.54_{-0.14}^{+0.42}$ & $0.353_{-0.003}^{+0.011}$ & \\
\hline
\end{tabular}

Notes. Error estimates are based on the normalized $\chi^{2}$ of the fits shown in Fig. 2, and on a 5\% uncertainty in the continuum levels. ${ }^{(a)}$ Analysis and error estimates inaccurate (velocity interval contains hot-core environment of unknown excitation, HF absorption is partially saturated). ${ }^{(b)}$ Nearand far-side crossing of Sagittarius spiral arm. ${ }^{(c)}$ Far-side crossing. ${ }^{(d)}$ Molecular hydrogen fractions $f_{\mathrm{H}_{2}}=2 N\left(\mathrm{H}_{2}\right) /\left(N(\mathrm{HI})+2 N\left(\mathrm{H}_{2}\right)\right)$ are derived from $\mathrm{HF}$ (with $N(\mathrm{HF}) / N\left(\mathrm{H}_{2}\right)=1.4 \times 10^{-8}$ ) and from $\mathrm{HI} \lambda 21 \mathrm{~cm}$ data, (Winkel et al. 2017, further references therein). ${ }^{(e)}$ Bayesian error estimates (accounting for the correlation between the column densities of the reported ratios).

apparent in Figs. 3 and 4. This variation is also a caveat against an overinterpretation of the stacking analysis.

Correlating the $\mathrm{CH}$ overabundance, defined as $0.4 N(\mathrm{CH}) / N(\mathrm{HF})$, with $N_{\mathrm{H}_{2}}$, or, for lack of it, with $N(\mathrm{HF})$ as substitute, indeed yields a fall-off of the $\mathrm{CH}$ overabundance with increasing column density - as expected if one associates larger column densities with larger volume densities, rather than with sightline crowding. The thus defined overabundance is shown in Fig. 6, against $1.65 \times 10^{11} \mathrm{~cm}^{-2}$ and $3.3 \times 10^{11} \mathrm{~cm}^{-2}$ wide HF column density intervals (for W49 N and W51 e2, respectively). We limit the analysis to column densities below the threshold where the bimodality becomes apparent. The width of the intervals is chosen so as to obtain a statistically meaningful result, as confirmed by the error bars derived from the variance of the overabundance within each interval. We selected only data points with at least $3 \sigma$ determinations of both $\mathrm{CH}$ and $\mathrm{HF}$ column densities, which excludes the portions of the HF spectrum affected by saturated absorption. The overabundances observed towards W49 N and W51 e2 level off from values of 3-4 to the canonical value of $\sim 1$ when the $\mathrm{H}_{2}$ column density increases by a factor of $\sim 5$. Interestingly, Godard et al. (2009), exploring the parameter space for the dissipation of turbulence, find similar ratios for a model with $A_{\mathrm{V}}=0.4$ (after correcting for the varying molecular hydrogen content), while at $A_{\mathrm{V}}=0.1$ the $\mathrm{CH}$ abundance varies only mildly. On the other hand, a single sightline contains diffuse cloud entities of markedly different characteristics, ranging from vortices embedded in a turbulent medium, with varying molecular content, to the precursors of molecular clouds. It seems fair to say that this statement holds throughout the whole Galaxy, not only in its central molecular zone, where these contrasting characteristics are much more pronounced (e.g., Ginsburg et al. 2016). Moreover, these manifestations of diffuse, cold gas may blend with each other even within a given spiral arm. Therefore, more spiral-arm crossings need to be investigated, including so far unexplored sightlines, because only solid statistical evidence can corroborate the theoretical work in a meaningful way.

Previous studies remain inconclusive regarding an overabundance of $\mathrm{CH}$. The dual-slope relationship between the column densities of $\mathrm{CO}$ and $\mathrm{CH}$, with a break close to $N(\mathrm{CH}) \sim 10^{13} \mathrm{~cm}^{-2}$, is attributed to increasing self-shielding of
Table 3. Best-fit parameters of the model for $\mathrm{CH}$ emission/absorption towards W3 IRS5.

\begin{tabular}{lcccc}
\hline \hline Layer & $v$ & $\Delta v$ & $N(\mathrm{CH})$ & $T_{\mathrm{ex}}$ \\
& {$\left[\mathrm{km} \mathrm{s}^{-1}\right]$} & {$\left[10^{14} \mathrm{~cm}^{-2}\right]$} & {$[\mathrm{K}]$} \\
\hline Core emission (HC) & -37.5 & 2.6 & 80.6 & 93.4 \\
& -34.9 & 4.3 & 62.6 & 133.9 \\
Common envelope (CE) & -39.7 & 5.4 & 2.4 & 25.7 \\
& -39.5 & 1.6 & 0.9 & 23.5 \\
Foreground screen (FG) & -38.1 & 9.6 & 4.8 & 64.1 \\
& -40.6 & 6.5 & 1.2 & 3.1 \\
& -39.5 & 2.0 & 0.8 & 3.1 \\
& +21.0 & 1.3 & 0.2 & 3.1 \\
& +0.5 & 2.7 & 0.1 & 3.1 \\
\hline
\end{tabular}

Notes. Cols. 2 and 3: center velocity and FWHM of Gauss-profile velocity components, respectively. Col. $4: \mathrm{d} N / \mathrm{d} v$ integrated across velocity profile of component. Components: HC - Emission components, radiatively pumped by hot core dust (beam-filling factor 0.003 ). $\mathrm{CE}$ - Three-component, moderately excited common envelope around emission components (beam-filling factor 0.15). FG - Cold foreground screen, seen in absorption in either line.

CO above the break point (Sheffer et al. 2008), and has nothing to do with a variation of the abundance of $\mathrm{CH}$.

The crossings of Galactic spiral arms are traced as clearly in $\mathrm{CH}$ as they are in HF (Table 2) and $\mathrm{OH}$ (Wiesemeyer et al. 2016). For instance, the contrast between the far-side crossing of the Sagittarius spiral arm and the adjacent interarm region in front of the Perseus arm is $\sim 10$ in $\mathrm{CH}$ and $\mathrm{HF}$, but only $\sim 2$ in molecular hydrogen fraction. This can be easily explained by the fact that $\mathrm{W} 49 \mathrm{~N}$ is located at a galactocentric distance of $7.6 \mathrm{kpc}$, where atomic gas is more abundant relative to molecular gas than on sightlines towards the inner Galaxy. A similar contrast has been found in $\mathrm{OH}$, which can be considered a secondary molecular hydrogen tracer (Gerin et al. 2016); Mookerjea (2016) indeed find no correlation of the $N(\mathrm{OH}) / N(\mathrm{CH})$ abundance ratio with the molecular hydrogen fraction, suggesting that both molecules trace $\mathrm{H}_{2}$ equally well, whatever proportion of the total hydrogen budget applies, and that the observed scatter in 


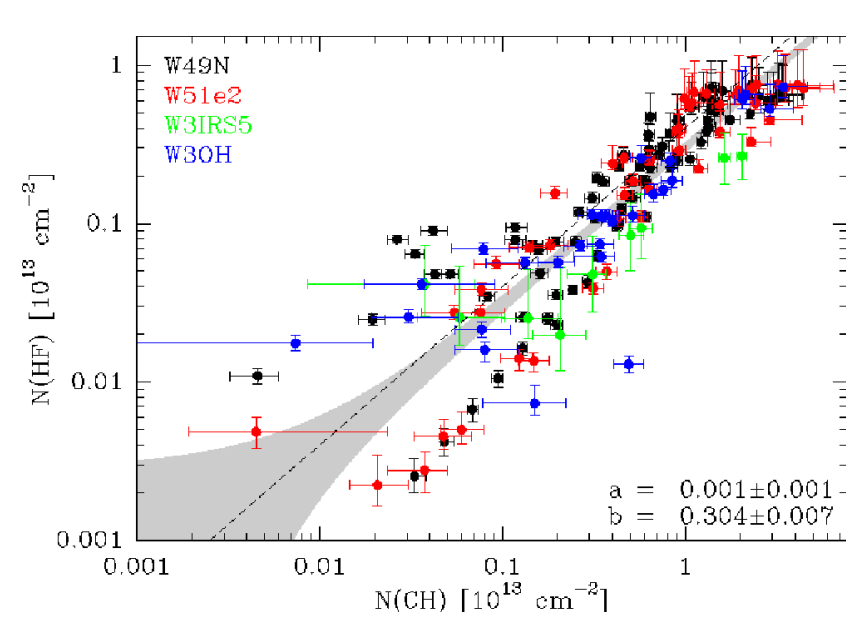

Fig. 4. As in Fig. 3, but for all data. A legend for the colors is given in the upper-left corner. The gray-shaded area covers all data points within the $3 \sigma$ limits of the weighted linear regression.

this abundance ratio is rather due to other factors, for example, a varying cosmic ray ionization rate. With our data we can confirm this conjecture: Fig. 5 shows the correlation between the column densities of $\mathrm{OH}$ and $\mathrm{CH}$ towards W49N (excluding velocity components where the entire $\mathrm{OH}$ absorption profile saturates). The correlation has a coefficient ( $r$-value) of 0.67 (falling below the $r$-value for the CH-HF correlations), a $p$-value of $1 \%$ and is significant (the Monte Carlo analysis detects a false alarm probability of $2.7 \%$ ). The weighted linear regression predicts an $\mathrm{OH} / \mathrm{CH}$ abundance ratio of $2.37 \pm 0.15$, which, for the Sheffer et al. (2008) relationship $N(\mathrm{CH}) / N\left(\mathrm{H}_{2}\right)=3.5 \times 10^{-8}$, converts to $N(\mathrm{OH}) / N\left(\mathrm{H}_{2}\right)=8.3 \times 10^{-8}$, reasonably close to both predicted and measured $\mathrm{OH}$ abundances (Albertsson et al. 2014; Wiesemeyer et al. 2016, respectively). Except for one outlier, the scatter in the $\mathrm{OH} / \mathrm{HF}$ abundance ratio is not larger than the scatter in the $\mathrm{CH} / \mathrm{HF}$. As a consequence, the over-abundance of $\mathrm{CH}$ at low column densities should also appear when, instead of $\mathrm{HF}, \mathrm{OH}$ is used as $\mathrm{H}_{2}$ tracer. This is indeed suggested by the distribution shown in Fig. 7 (totally absorbed velocity components are not considered in this analysis, as in the case of $\mathrm{HF})$. However, owing to the larger errors, the evidence is less compelling. As a reminder, we recall that even within a narrow velocity interval all correlated quantities are averages of diffuse and translucent cloud entities along the sightline, exposed to varying conditions. As a matter of fact, the abundances of $\mathrm{CH}$, $\mathrm{OH}$, and $\mathrm{H}_{2} \mathrm{O}$ are particularly sensitive to the cosmic ray ionization rate (Godard et al. 2014). One may also speculate that the correlation between $\mathrm{CH}$ and $\mathrm{OH}$ is less pronounced than that between $\mathrm{CH}$ and HF because at low densities $\left(n_{\mathrm{H}} \lesssim 30 \mathrm{~cm}^{-3}\right)$ $\mathrm{OH}$ has a production channel in the relaxation stage following a burst of turbulent dissipation, as shown by Godard et al. (2014): while the $\mathrm{OH}$ abundance falls below that of $\mathrm{CH}$ immediately after the burst, the $\mathrm{OH} / \mathrm{CH}$ exceeds unity during the subsequent relaxation stage, on a timescale that depends on the dissipated energy per unit length. It is interesting to note that Liszt \& Lucas (2002) determine (across a limited $N\left(\mathrm{H}_{2}\right)$ range) a ratio of $N(\mathrm{OH}) / N(\mathrm{CH})=3.0 \pm 0.9$, in good agreement with our value derived from the $\mathrm{W} 49 \mathrm{~N}$ sightline (in their study, the uncertain microwave-derived $\mathrm{CH}$ column densities were augmented by optical measurements). Even more remarkable is their significant correlation between the microwave $\mathrm{CH}$ and $\mathrm{OH}$ emission-line areas; the data from the sightline to $\zeta$ Oph seem to suggest a bimodal distribution of $\mathrm{CH}$ at low $\mathrm{OH}$ column densities. From a compilation of four sightlines and two dark clouds

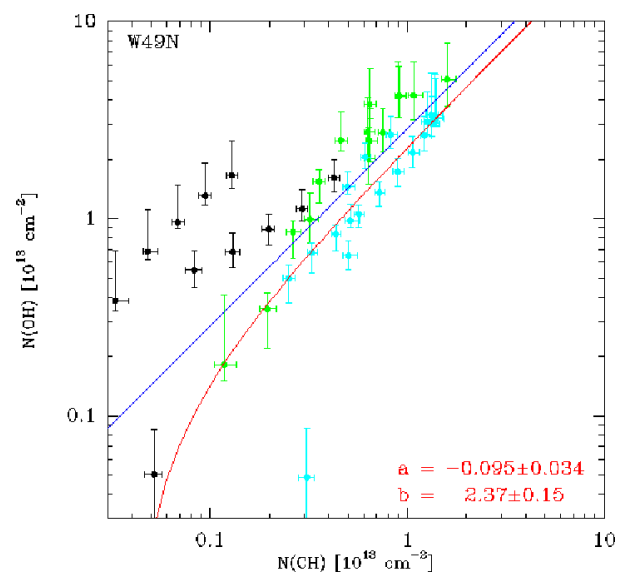

Fig. 5. Correlation between $\mathrm{OH}$ and $\mathrm{CH}$ column densities towards W49 $\mathrm{N}$. The blue line is for a $N(\mathrm{OH}) / N\left(\mathrm{H}_{2}\right)$ of $10^{-7}$. The red line shows the weighted linear regression, the resulting $\mathrm{OH} / \mathrm{CH}$ abundance ratio is given in the lower-right corner. Velocity bins for the column densities and color-codes are as in Fig. 3.

they conclude that when the main gas-phase carrier of carbon is $\mathrm{CO}$ rather than $\mathrm{C}^{+}$, the $\mathrm{CH}$ abundance declines markedly, in contrast to that of $\mathrm{OH}$. This may be another indication of a bimodal or dual-slope relationship between $N(\mathrm{CH})$ and $N\left(\mathrm{H}_{2}\right)$.

We conclude this section with a remarkable detail observed on the sightlines to $\mathrm{W} 3(\mathrm{OH})$ and W3 IRS5. Although the illuminating hot cores are separated by only 16!6 (9.7 pc), the column density profiles are quite different, even in the local arm where only the sightline to $\mathrm{W} 3(\mathrm{OH})$ sees a narrow, $\sim 1 \mathrm{~km} \mathrm{~s}^{-1}$-wide feature (unlike the sightline to $\mathrm{W} 49 \mathrm{~N}$, there is no risk of confusion with other spiral arms close to zero velocity). Assuming a length of at most $500 \mathrm{pc}$ for the path through the local arm (Lallement et al. 2014), this converts to an upper limit of $2.4 \mathrm{pc}$ for the diffuse cloud size. A similar upper limit was estimated by Winkel et al. (2017) for the sightline to SgrB2 M/N. Interestingly, Liszt \& Gerin (2016) determine a clumping scale of $5.5 \mathrm{pc}$ for the gas on the sightline to W 31C. It seems fair to say that these estimates refer to the largest scales in diffuse gas entities, with presumably considerable sub-structure.

\section{2. $\mathrm{CH}$ as molecular gas tracer in environments forming high-mass stars}

The following section demonstrates the usefulness of the $\mathrm{CH}$ ground state transitions in the high-density environments associated with sites of high-mass star formation. Because of the saturated absorption in $\mathrm{HF}$ and $\mathrm{OH}$ towards the background sources, only the optically thin line wings of these tracers are usable as tools for measuring the $\mathrm{H}_{2}$ column density in the star-forming environment, provided that they do not blend with unrelated foreground gas. As already mentioned, the far-infrared lines of $\mathrm{CH}$ do not suffer from this drawback, and the combined analysis of the $\lambda 149 \mu \mathrm{m}$ and $\lambda 560 \mu \mathrm{m}$ lines allows one to further constrain the physical conditions characterizing the emission region. For $\mathrm{OH}$, such an approach was demonstrated by Csengeri et al. (2012). In the following we try to extract a maximum of quantities directly from the sub-millimeter/far-infrared spectroscopy of $\mathrm{CH}$, with an indispensable minimum of assumptions. For the far-infrared transition, only one lambda doublet component is available. Because the spectroscopic properties of both components are not very different (Table 1), this does not result in a major lack of information; fitting both components 

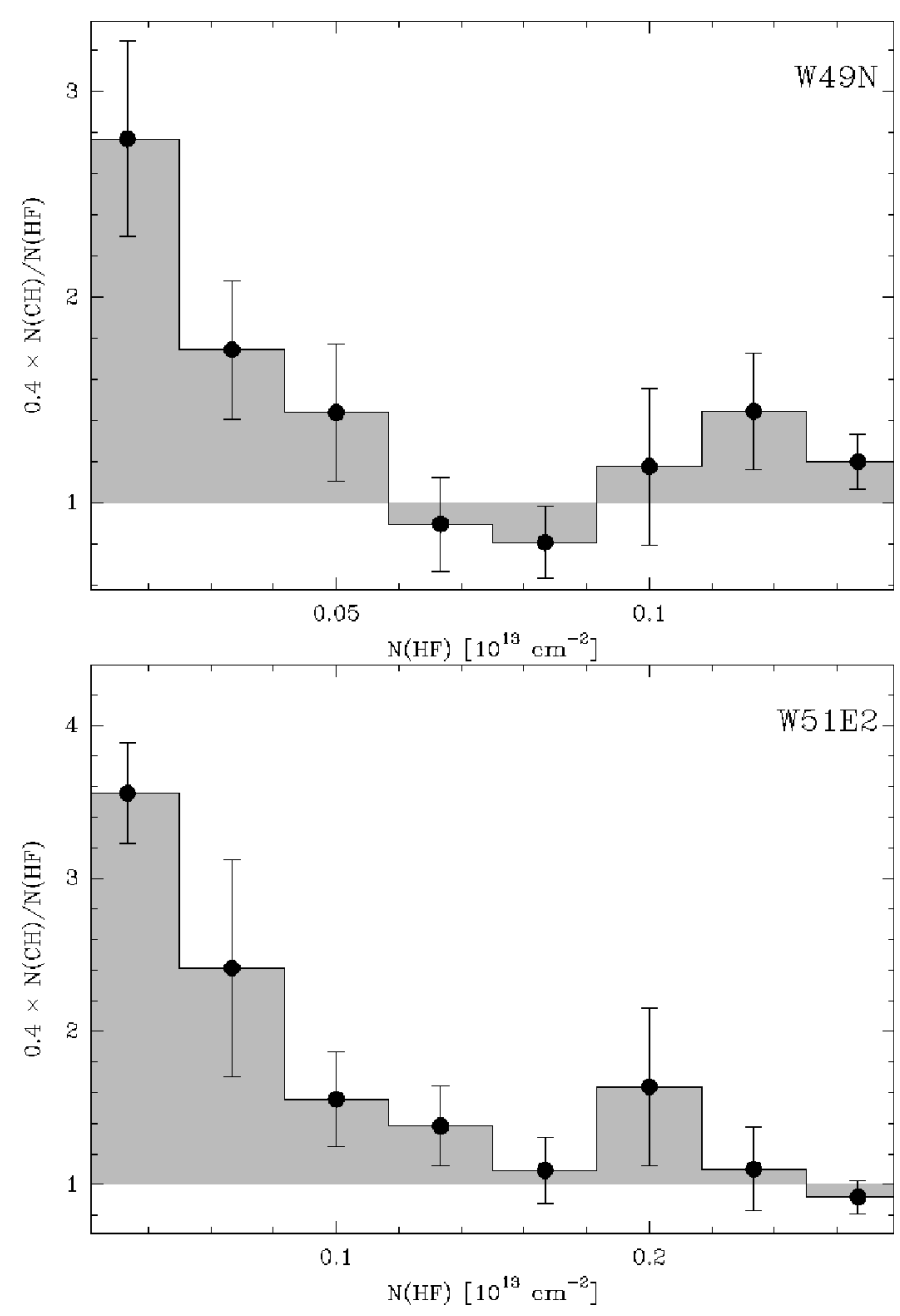

Fig. 6. Over-abundance of $\mathrm{CH}$, defined as $0.4 N(\mathrm{CH}) / N(\mathrm{HF})$ vs. $N(\mathrm{HF})$. Column densities are derived from $1 \mathrm{~km} \mathrm{~s}^{-1}$-wide velocity intervals in the spectral distribution of $N_{\mathrm{v}}$ (cf. Fig. 2). Only column densities with at least a $3 \sigma$ detection are used. Error-bars are deduced from the variances of the abundances within each histogram bin.

merely represents an additional consistency check for the derived column density of $\mathrm{CH}$. Among the targets presented above, we select W3 IRS5. Its location at a distance of $2.0 \mathrm{kpc}$ in the second quadrant of the Galaxy in the Perseus arm avoids the confusion arising in the $\lambda 560 \mu \mathrm{m}$ line owing to its large hyperfine splitting and leading to a blend of emission and absorption components originating from different locations on the sightline.

W3 IRS5 is a bright infrared source discovered by Wynn-Williams et al. (1972) and harbors a cluster of hypercompact HII regions (e.g., Wilson et al. 2003; van der Tak \& Menten 2005) powered by five proto-OB stars (Megeath et al. 2005), which lend the object the designation of a "Trapezium in its making" by the latter authors. The dust continuum emission of its components SMM1 to SMM5 was analyzed by Wang et al. (2013); they derive column densities of $N\left(\mathrm{H}_{2}\right)=$ $1.0-4.4 \times 10^{23} \mathrm{~cm}^{-2}$, assuming a dust temperature of $150 \mathrm{~K}$ and source diameters of $\sim 1^{\prime \prime}$ (confirmed by a visibility analysis of their Submillimeter Array (SMA) data, see also Wilson et al. 2003).

Our model consists of an ensemble of hot cores (labeled $h c$ in the following), corresponding to SMM1 to SMM5, a common envelope (ce) associated with the entire W3 IRS5 complex, and a foreground screen $(f g)$ representing the Perseus spiral arm

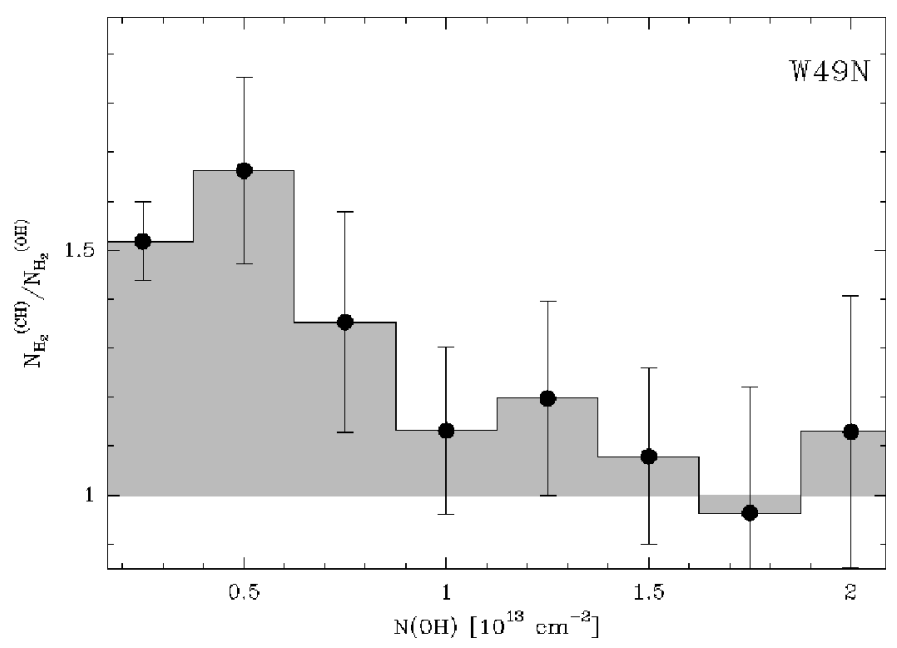

Fig. 7. As in Fig. 6, but using $\mathrm{OH}$ as $\mathrm{H}_{2}$ proxy instead of HF. The ordinate shows the ratio between $\mathrm{H}_{2}$ column densities derived from $\mathrm{CH}$ and those derived from $\mathrm{OH}$ (assuming an $\mathrm{OH} / \mathrm{H}_{2}$ abundance of $10^{-7}$ ).

and gas in the solar neighborhood. The objective function of the model is given by

$\chi^{2}=\left\langle w_{\lambda 560}\left(T_{\lambda 560}^{(\mathrm{mod})}-T_{\lambda 560}^{(\mathrm{obs})}\right)^{2}+w_{\lambda 149}\left(T_{\lambda 149}^{(\mathrm{mod})}-T_{\lambda 149}^{(\mathrm{obs})}\right)^{2}\right\rangle$,

with (omitting the indices $\lambda 149, \lambda 560$ )

$T^{(\text {mod })}=\left\{\Phi_{\mathrm{hc}}\left(\Sigma_{\mathrm{c}}+\Sigma_{\mathrm{hc}}\left(1-\mathrm{e}^{-\tau_{\mathrm{hc}}}\right)\right) \mathrm{e}^{-\tau_{\mathrm{ce}}}+\Phi_{\mathrm{ce}} \Sigma_{\mathrm{ce}}\left(1-\mathrm{e}^{-\tau_{\mathrm{ce}}}\right)\right\} \mathrm{e}^{-\tau_{\mathrm{fg}}}$,

where $T^{(\mathrm{mod})}$ and $T^{(\mathrm{obs})}$ represent the modeled and observed brightness temperature of either line, respectively. The average is to be taken across the spectral bandpass of the full line profiles; $w_{\lambda 560}$ and $w_{\lambda 149}$ are weight factors accounting for the individual signal-to-noise ratio in each transition. $\Phi_{\mathrm{hc}}$ and $\Phi_{\mathrm{ce}}$ are the beamfilling factors of the hot core and common envelope, respectively (assuming that the hot dust continuum and the hot-core emission fill the same beam fractions). The source functions $\Sigma_{\mathrm{c}}$ for the continuum emission, and $\Sigma_{\mathrm{hc}}$ and $\Sigma_{\mathrm{ce}}$ for the hot core and common envelope line emission, respectively, are expressed as Rayleigh-Jeans temperatures. We note that in Eq. (4) the source functions are expressed as unique, equivalent excitation temperatures, related to the actual source function profile along the sightline, $\Sigma(z)$, by

$T_{\text {ex,eqv }}=\frac{\int_{0}^{\mathrm{L}} \Sigma(z) \kappa(z) \mathrm{e}^{-\int_{z}^{\mathrm{L}} \kappa\left(z^{\prime}\right) \mathrm{d} z^{\prime}} \mathrm{d} z}{1-\mathrm{e}^{-\int_{0}^{\mathrm{L}} \kappa(z) \mathrm{d} z}}$,

where $L$ is the length of the sightline through the source. We emphasize that a unique emissivity is not required for Eq. (4) to be valid, that is, the opacity coefficient $\kappa$ of a given component does not need to be constant. While the width and center velocities of the Gaussian decomposition of the $\mathrm{d} N / \mathrm{d} v$ profile are well constrained by the $\lambda 149 \mu \mathrm{m}$ absorption spectrum, the degeneracy between the filling factors and excitation temperatures can only be lifted by the dependence of the optical depths on the excitation. As shown in Fig. C.1, the $\lambda 560 \mu \mathrm{m}$ line is more sensitive to the excitation than the line at $\lambda 149 \mu \mathrm{m}$. For a least-square fit to both $\mathrm{CH}$ lines, we apply the Metropolis algorithm (Metropolis et al. 1953, see also Press et al. 1992 for an extension to continuous minimization). A straightforward implementation of the algorithm yields a satisfactory fit, although the convergence is 


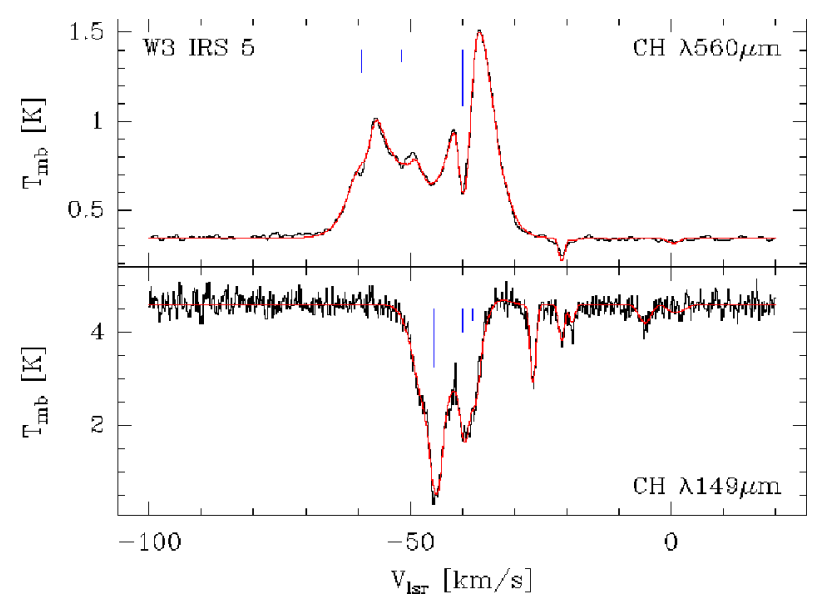

Fig. 8. $\mathrm{CH}$ emission and absorption towards W3 IRS5. Top panel: $\lambda 560 \mu \mathrm{m}$ line (HIFI/PRISMAS). Bottom panel: $\lambda 149 \mu \mathrm{m}$ (upGREAT). The red lines indicate the common fit to both spectra (in the $\chi^{2}$ sense). The hyperfine splitting is indicated by blue markers, scaling with the corresponding weight for a complete ground-state level population. The strongest hyperfine component of the $\lambda 560 \mu \mathrm{m}$ line is centered close to the systemic velocity. For details see text.

slow. When the convergence became quasi-linear, it was possible to accelerate it by minimization of residua (Auer 1987). The result is shown in Fig. 8, and the best-fit parameters are listed in Table 3. The normalized $\chi^{2}$ of our fit falls below $1 \%$, a result which could only be achieved by assigning three components to the warm, common envelope (likewise, various $\mathrm{H}_{2} \mathrm{O}$ lines observed with HIFI were easier to fit with a double component model, Chavarría et al. 2010). These components appear in absorption against two highly excited emission components (presumably co-spatial with the hot core dust), otherwise in emission. The prominent self-absorption at $-40 \mathrm{~km} \mathrm{~s}^{-1}$, in the strongest hyperfine-component of the $\lambda 560 \mu \mathrm{m}$ line, coincides with the radial velocity of the $\mathrm{H}_{2} \mathrm{O}$ masers at $-40.7 \pm 1.3 \mathrm{~km} \mathrm{~s}^{-1}$ (Imai et al. 2000, their group A outflow). Rather than treating the beam-filling factors in Eq. (4) as additional free parameters in a high-dimensional parameter space, we fix them by means of ancillary continuum data: identifying the aforementioned compact, $\sim 1^{\prime \prime}$-wide dust sources in the SMA continuum image (Wang et al. 2013) with our hot emission components, the filling factor for the 39.'5 -wide HIFI beam (FWHM) amounts to $\Phi_{\mathrm{hc}}=0.003$. For the moderately warm common envelope, the SMA data are less reliable for an estimate of $\Phi_{\text {ce }}$, not least due to the lack of short spatial frequencies in the continuum visibilities. We follow Chavarría et al. (2010) and use a $\lambda 800 \mu \mathrm{m}$ single-dish continuum map (Oldham et al. 1994), allowing us to estimate a beam-deconvolved size of $\simeq 15^{\prime \prime}$, which converts to a beam filling of $\Phi_{\mathrm{ce}}=0.15$. Finally, two cold foreground components were needed for a satisfactory fit. Given their velocities, they are probably related to the outer layers of the common envelope. The narrower component (at $-39.5 \mathrm{~km} \mathrm{~s}^{-1}$ ) coincides with a common envelope component of comparable width. This suggests that both components belong to the same structure, characterized by a negative excitation gradient. Replacing these two components by a single one increases the normalized $\chi^{2}$ by $6 \%$ and yields an unsatisfactory fit in the $(-50,-40) \mathrm{km} \mathrm{s}^{-1}$ velocity interval, regardless of whether this component is located in the common envelope or the cold foreground gas. As a matter of fact, we note that around $50 \mathrm{~km} \mathrm{~s}^{-1}$ the fit to the $\lambda 560 \mu \mathrm{m}$ line is still imperfect, even with the nine components ultimately retained. Two further components at

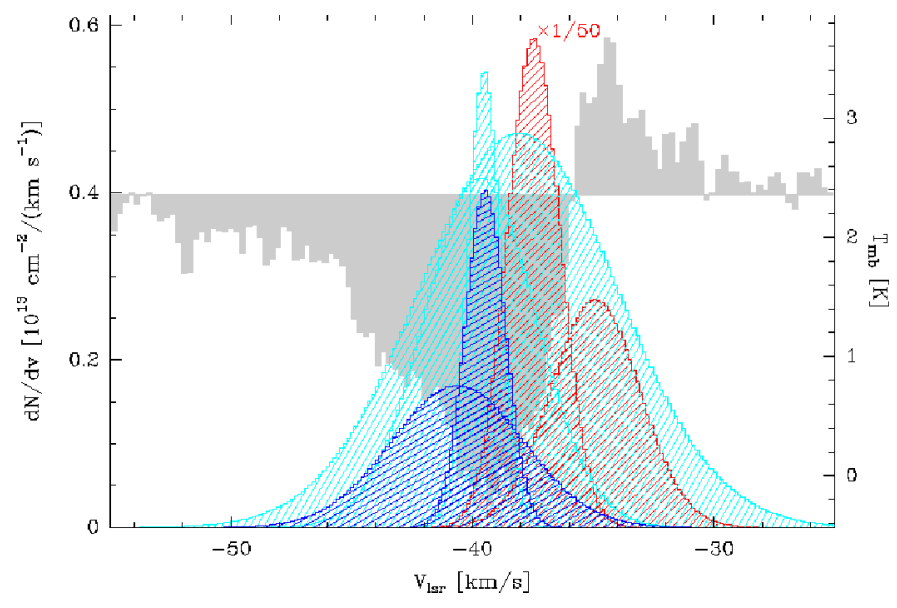

Fig. 9. Breakup of $\mathrm{CH}$ components observed around W3 IRS5. Red: $\mathrm{d} N / \mathrm{d} v$ for the two hot core components (scaled down by a factor of 50). Cyan: same for the three common-envelope components. Blue: cold foreground components. The gray histogram shows the P Cygni profile of the HF ground-state transition $(J=1 \rightarrow 0$, archival HIFI data, PRISMAS key science project). For details see text and Table 3.

$v_{\text {lsr }}=0.5$ and $-21 \mathrm{~km} \mathrm{~s}^{-1}$ are unrelated to W3 IRS5 and can be identified with local gas and with the near-side boundary of the Perseus spiral arm, respectively. Thus, rather than freely fitting these two components, we kept their parameters fixed, adopting the values from the analysis described in Sect. 4.1.

Evidently, only a dedicated non-LTE simulation can explain the fitted excitation temperatures as a function of the physical conditions encountered in the hot core and common envelope components. While this is beyond the scope of this paper, we conclude this discussion with a comparison with the P Cygnitype profile seen in the HF $J=1 \rightarrow 0$ line and other light hydrides (Benz et al. 2010). Figure 9 shows the column density profiles for the various components against the HF spectrum. While the dominant contribution to the total column density originates from the two hot core components, the prominent, saturated absorption feature seen in HF is due to the same low-excitation material in the common envelope and foreground that is also traced by the absorption seen in the $\mathrm{CH}$ lines. Our component separation clearly demonstrates that the bulk of this absorption occurs at velocities that are blue-shifted with respect to the emission components. The driving agent for the expansion of the common envelope is most likely a system of multiple outflows (Rodón et al. 2008). Another hint that in such an environment $\mathrm{CH}$ remains a reliable tracer for molecular hydrogen is provided by the comparison of the derived column densities with corresponding estimates using the $\lambda 850 \mu \mathrm{m}$ dust emission (Wang et al. 2013): the $\mathrm{H}_{2}$ column densities in the two emission components amount to 2.3 and $1.8 \times 10^{23} \mathrm{~cm}^{-2}$ (for $\left.N(\mathrm{CH}) / N\left(\mathrm{H}_{2}\right)=3.5 \times 10^{-8}\right)$, which agree within a factor 2 with those derived from the emission from the two dominant dustcontinuum components (sources SMM1 and SMM2 in Wang et al. 2013). However, this good agreement may be a chance coincidence: we have no proof that the best-fit solution summarized in Table 3 is unique. The same holds for the rough dust-mass estimates (which, instead of a full continuum radiative transfer, assume an ad-hoc dust temperature of $150 \mathrm{~K}$ ).

\section{Conclusions}

We close this work with a summary of its main results:

1. For diffuse and translucent interstellar clouds, we confirm a prominent, but not very tight correlation between the column 
densities of $\mathrm{CH}$ and $\mathrm{HF}$. While a ratio of $N(\mathrm{CH}) / N(\mathrm{HF}) \simeq$ 0.4 represents rather a boundary to the observed relationship, a bimodal distribution of $\mathrm{CH}$ (with respect to $\mathrm{HF}$ ) was found below $N(\mathrm{CH}) \lesssim 10^{13} \mathrm{~cm}^{-2}$ (i.e., $N\left(\mathrm{H}_{2}\right) \simeq 3 \times 10^{20} \mathrm{~cm}^{-2}$ or $\left.A_{\mathrm{V}} \lesssim 0.3\right)$. We identify the lower branch of this distribution with the action of the endothermic reaction pathway, forming $\mathrm{CH}^{+}$thanks to the molecular hydrogen abstraction reaction $\mathrm{C}^{+}\left(\mathrm{H}_{2}, \mathrm{H}\right) \mathrm{CH}^{+}$. The subsequent, fast hydrogen abstraction reaction forms $\mathrm{CH}_{2}^{+}$, which is a prerequisite to form $\mathrm{CH}$ via dissociative recombination: $\mathrm{CH}^{+}\left(\mathrm{H}_{2}, \mathrm{H}\right) \mathrm{CH}_{2}^{+}\left(\mathrm{e}^{-}, \mathrm{H}\right) \mathrm{CH}$ Because the alternative pathway (forming $\mathrm{CH}_{2}^{+}$by a slow radiative association of $\mathrm{C}^{+}$with $\mathrm{H}_{2}$ ) is less efficient, the former pathway efficiently boosts the $\mathrm{CH}$ abundance. Our finding that this over-abundance of $\mathrm{CH}$ appears in the lowerdensity regime strongly suggests that the agent to overcome the activation barrier of $4640 \mathrm{~K}$ is dissipation of turbulence. Using $\mathrm{OH}$ instead of $\mathrm{HF}$ as a $\mathrm{H}_{2}$ proxy yields the same conclusion (albeit at lower significance). By the same token we determine an $\mathrm{OH} / \mathrm{CH}$ abundance ratio of $2.37 \pm 0.15$.

2 . In the envelopes surrounding cores forming high-mass stars, the combined analysis of the $\lambda 149 \mu \mathrm{m}$ and $\lambda 560 \mu \mathrm{m}$ ground state transitions of $\mathrm{CH}$ allows to separate emission features from absorbing layers in the second transition, and therefore to better constrain both the column density and excitation of $\mathrm{CH}$ in such environments. Follow-up studies will include a better determination of the beam-filling factors for the far-infrared/sub-millimeter observations, by means of radio interferometry of the $3 \mathrm{GHz}$ transitions between the hyperfine components in the ground state. While these lines, fraught with non-LTE effects such as population inversion, will be difficult to interpret without reliable collision coefficients at hand, radiative transfer models for the farinfrared/sub-millimeter lines would be the next step in the analysis, for which this work may provide suitable boundary conditions.

Acknowledgements. Based in part on observations made with the NASA/DLR Stratospheric Observatory for Infrared Astronomy. SOFIA Science Mission Operations are conducted jointly by the Universities Space Research Association, Inc., under NASA contract NAS2-97001, and the Deutsches SOFIA Institut under DLR contract 50 OK 0901 . We gratefully acknowledge the support by the observatory staff. An anonymous referee provided valuable comments which improved the paper.

\section{References}

Agúndez, M., Goicoechea, J. R., Cernicharo, J., Faure, A., \& Roueff, E. 2010, ApJ, 713, 662

Albertsson, T., Indriolo, N., Kreckel, H., et al. 2014, ApJ, 787, 44

Auer, L. H. 1987, Acceleration of Convergence, in Numerical Radiative Transfer, ed. W. Kalkofen (Cambridge: Cambridge University Press), 101

Barlow, R. 2003, ArXiv e-prints [arXiv:physics/0306138]

Benz, A. O., Bruderer, S., van Dishoeck, E. F., et al. 2010, A\&A, 521, L35

Black, J. H., \& Dalgarno, A. 1977, ApJS, 34, 405

Black, J. H., Dalgarno, A., \& Oppenheimer, M. 1975, ApJ, 199, 633

Capriotti, E. R. 1965, ApJ, 142, 1101

Chavarría, L., Herpin, F., Jacq, T., et al. 2010, A\&A, 521, L37

Csengeri, T., Menten, K. M., Wyrowski, F., et al. 2012, A\&A, 542, L8

Douglas, A. E., \& Herzberg, G. 1941, ApJ, 94, 381

Draine, B. T. 1978, ApJS, 36, 595

Dunham, Jr., T. 1937, PASP, 49, 26

Elitzur, M., \& Asensio Ramos, A. 2006, MNRAS, 365, 779

Falgarone, E., Pety, J., \& Hily-Blant, P. 2009, A\&A, 507, 355

Falgarone, E., Godard, B., Cernicharo, J., et al. 2010a, A\&A, 521, L15

Falgarone, E., Ossenkopf, V., Gerin, M., et al. 2010b, A\&A, 518, L118

Faure, A., Halvick, P., Stoecklin, T., et al. 2017, MNRAS, 469, 612

Galván-Madrid, R., Liu, H. B., Zhang, Z.-Y., et al. 2013, ApJ, 779, 121

Gerin, M., de Luca, M., Goicoechea, J. R., et al. 2010, A\&A, 521, L16

Gerin, M., Levrier, F., Falgarone, E., et al. 2012, Phil. Trans. R. Soc. London, Ser. A, 370,5174
Gerin, M., Ruaud, M., Goicoechea, J. R., et al. 2015, A\&A, 573, A30 Gerin, M., Neufeld, D. A., \& Goicoechea, J. R. 2016, ARA\&A, 54, 181 Gerlich, D., Disch, R., \& Scherbarth, S. 1987, J. Chem. Phys., 87, 350 Ginsburg, A., Henkel, C., Ao, Y., et al. 2016, A\&A, 586, A50

Ginsburg, A., Goddi, C., Kruijssen, J. M. D., et al. 2017, ApJ, 842, 92

Godard, B., Falgarone, E., \& Pineau Des Forêts, G. 2009, A\&A, 495, 847 Godard, B., Falgarone, E., \& Pineau Des Forêts, G. 2014, A\&A, 570, A27 Gough, S., Schermann, C., Pichou, F., et al. 1996, A\&A, 305, 687 Guan, X., Stutzki, J., Graf, U. U., et al. 2012, A\&A, 542, L4 Hachisuka, K., Brunthaler, A., Menten, K. M., et al. 2006, ApJ, 645, 337 Hierl, P. M., Morris, R. A., \& Viggiano, A. A. 1997, J. Chem. Phys., 106, 10145 Hirsch, L., Adams, J. D., Herter, T. L., et al. 2012, ApJ, 757, 113 Imai, H., Kameya, O., Sasao, T., et al. 2000, ApJ, 538, 751

Indriolo, N., Neufeld, D. A., Seifahrt, A., \& Richter, M. J. 2013, ApJ, 764, 188

Kester, D., Higgins, R., \& Teyssier, D. 2017, A\&A, 599, A115

Klein, B., Hochgürtel, S., Krämer, I., et al. 2012, A\&A, 542, L3

Lallement, R., Vergely, J.-L., \& Puspitarini, L. 2014, Mem. Soc. Astron. It., 85, 339

Levrier, F., Le Petit, F., Hennebelle, P., et al. 2012, A\&A, 544, A22

Liszt, H., \& Lucas, R. 2002, A\&A, 391, 693

Liszt, H. S., \& Gerin, M. 2016, A\&A, 585, A80

Liu, T., Kim, K.-T., Wu, Y., et al. 2015, ApJ, 810, 147

Marinakis, S., Dean, I. L., Kłos, J., \& Lique, F. 2015, Phys. Chem. Chem. Phys., 17,21583

Mathis, J. S., Mezger, P. G., \& Panagia, N. 1983, A\&A, 128, 212

Megeath, S. T., Wilson, T. L., \& Corbin, M. R. 2005, ApJ, 622, L141

Megeath, S. T., Townsley, L. K., Oey, M. S., \& Tieftrunk, A. R. 2008, in Handbook of Star Forming Regions, I: The Northern Sky, ed. B. Reipurth (ASP Monograph Publications), 4, 264

Metropolis, N., Rosenbluth, A. W., Rosenbluth, M. N., Teller, A. H., \& Teller, E. 1953, J. Chem. Phys., 21, 1087

Monje, R. R., Emprechtinger, M., Phillips, T. G., et al. 2011, ApJ, 734, L23

Mookerjea, B. 2016, MNRAS, 459, 2822

Neufeld, D. A., Zmuidzinas, J., Schilke, P., \& Phillips, T. G. 1997, ApJ, 488, L141

Neufeld, D. A., Wolfire, M. G., \& Schilke, P. 2005, ApJ, 628, 260

Naylor, D. A., Dartois, E., Habart, E., et al. 2010, A\&A, 518, L117

Oka, T., Welty, D. E., Johnson, S., et al. 2013, ApJ, 773, 42

Oldham, P. G., Griffin, M. J., Richardson, K. J., \& Sandell, G. 1994, A\&A, 284, 559

Pan, K., Federman, S. R., Sheffer, Y., \& Andersson, B.-G. 2005, ApJ, 633, 986

Parikka, A., Habart, E., Bernard-Salas, J., et al. 2017, A\&A, 599, A20

Pickett, H. M., Poynter, R. L., Cohen, E. A., et al. 1998, J. Quant. Spectr. Rad. Transf., 60, 883

Pineau des Forets, G., Flower, D. R., Hartquist, T. W., \& Dalgarno, A. 1986, MNRAS, 220, 801

Press, W. H., Teukolsky, S. A., Vetterling, W. T., \& Flannery, B. P. 1992, Numerical Recipes in C. The Art of Scientific Computing (Cambridge: Cambridge University Press)

Pütz, P., Honingh, C. E., Jacobs, K., et al. 2012, A\&A, 542, L2

Qin, S.-L., Schilke, P., Wu, J., et al. 2016, MNRAS, 456, 2681

Reid, M. J., Menten, K. M., Brunthaler, A., et al. 2014, ApJ, 783, 130

Risacher, C., Güsten, R., Stutzki, J., et al. 2016, A\&A, 595, A34

Rodón, J. A., Beuther, H., Megeath, S. T., \& van der Tak, F. F. S. 2008, A\&A, 490, 213

Salgado, F., Berné, O., Adams, J. D., et al. 2012, ApJ, 749, L21

Sato, M., Reid, M. J., Brunthaler, A., \& Menten, K. M. 2010, ApJ, 720, 1055

Sellke, T., Bayarri, M. J., \& Berger, J. O. 2001, Am. Stat., 55, 62

Sheffer, Y., Rogers, M., Federman, S. R., et al. 2008, ApJ, 687, 1075

Snow, T. P., \& McCall, B. J. 2006, ARA\&A, 44, 367

Sonnentrucker, P., Wolfire, M., Neufeld, D. A., et al. 2015, ApJ, 806, 49

Stacey, G. J., Lugten, J. B., \& Genzel, R. 1987, ApJ, 313, 859

Swings, P., \& Rosenfeld, L. 1937, ApJ, 86, 483

Vallée, J. P. 2008, AJ, 135, 1301

van der Tak, F. F. S., \& Menten, K. M. 2005, A\&A, 437, 947

Voshchinnikov, N. V., Semenov, D. A., \& Henning, T. 1999, A\&A, 349, L25

Wang, K.-S., Bourke, T. L., Hogerheijde, M. R., et al. 2013, A\&A, 558, A69

Wiesemeyer, H., Güsten, R., Heyminck, S., et al. 2016, A\&A, 585, A76

Wilson, T. L., Boboltz, D. A., Gaume, R. A., \& Megeath, S. T. 2003, ApJ, 597, 434

Winkel, B., Wiesemeyer, H., Menten, K. M., et al. 2017, A\&A, 600, A2

Wynn-Williams, C. G., Becklin, E. E., \& Neugebauer, G. 1972, MNRAS, 160, 1

Xu, Y., Reid, M. J., Zheng, X. W., \& Menten, K. M. 2006, Science, 311, 54

Zanchet, A., Godard, B., Bulut, N., et al. 2013, ApJ, 766, 80

Zhang, B., Reid, M. J., Menten, K. M., et al. 2013, ApJ, 775, 79

Zhu, C., Krems, R., Dalgarno, A., \& Balakrishnan, N. 2002, ApJ, 577, 795 


\section{Appendix A: Error estimates for double-sideband receiving systems}

The determination of column densities from absorption spectra is straightforward. This holds also for the case of hyperfinesplit spectra where the total column densities can be derived either by least-square fitting of a correspondingly weighted target function, or by direct deconvolution (Gerin et al. 2010; Jacob et al., in prep.). Nevertheless, a candid discussion of measurement errors is essential. This statement holds in particular for a double-sideband receiving system, where the calibration of the continuum level is fraught with uncertainties arisi ng from, for example, the atmospheric transmission or the signal-to-image band gain ratio. The measurement equation reads

$T=T_{\mathrm{c}} \exp (-\tau)+\Delta T_{\mathrm{c}}$,

where $\Delta T_{\mathrm{c}}$ is an unknown contribution from the image band. While the variance of $T_{\mathrm{c}}$ can be guessed from the unnormalized $\chi^{2}$ of a least-square fit, $\Delta T_{\mathrm{c}}$ is a priori unknown, which is why the target function is assumed to read

$T=\left(T_{\mathrm{c}}+\Delta T_{\mathrm{c}}\right) \exp (-(\tau+\Delta \tau))$,

where $\Delta \tau$ is the error resulting from the wrong attribution of $\Delta T_{\mathrm{c}}$ to the signal band. Introducing $r=\Delta T_{\mathrm{c}} / T_{\mathrm{c}}$, one finds from the above equations

$\Delta \tau=\log (1+r)-\log (r+\exp (-\tau))-\tau$,

which is converted into a column density error thanks to Eq. (1). The Bayesian error estimates throughout this paper are based on Monte Carlo simulations assuming Gaussian distributions of $T_{\mathrm{c}}$ (with $\sigma^{2}=\chi^{2}$ ) and $\Delta T_{\mathrm{c}}$. From a closer inspection of the atmospheric transmission in the signal and image band of the $\lambda 149 \mu \mathrm{m}$ line, and assuming typical calibration errors, we use a Gaussian distribution of $\Delta T_{\mathrm{c}}$ with $\sigma_{\mathrm{r}}=0.05 T_{\mathrm{c}}$. The assessment of the resulting asymmetric errors will be discussed in the next section.

Our assumption of a $5 \%$ uncertainty (r.m.s.) in the continuum level is motivated by aspects related to hardware performance, and to the origin and transmission of the intrinsic continuum emission. As for the hardware, hot electron bolometer mixers (e.g., Pütz et al. 2012) are characterized by signal-to-image band gain ratios close to unity. Kester et al. (2017) provide a comprehensive study, showing that for HIFI bands 6 and 7, using the same technology as upGreat, the uncertainty in the gain ratio falls below 4\%. At this accuracy, there is no evidence for a departure from the theoretically expected 1:1 gain ratio. Even a significant departure would not necessarily entail a corresponding calibration error as long as the spectral index of the continuum emission remains sufficiently small. The rest frequency of the far-infrared ground-state transition of $\mathrm{CH}$ falls below the powerlaw part and the maximum of the spectral energy distribution of $\sim 150 \mathrm{~K}$ hot dust. For dust opacity indices of 1.0 (expected for a predominant population of large dust grains) and 2.0, the relative difference in the continuum emission in the signal and image band amounts to 0.5 and $0.7 \%$, respectively, for the applied sideband separations of 3.4 and $3.6 \mathrm{GHz}$ (for W49 N/W51 e2, and W3 OH/W3 IRS5, respectively). The coupling efficiencies of the upGreat pixels are monochromatic across the frequency range framed by the sidebands. Under the observing conditions described in Sect. 2, the relative difference in the atmospheric signal and image band transmissions amounts to at most $1 \%$. Quadratically adding the quoted errors yields a $4.2 \%$ uncertainty, falling short of the therefore conservative, retained 5\% level. For the HF $J=1 \rightarrow 0$ line, which was observed in HIFI band 5, a similar error estimate applies, despite the different technology (SIS mixers, see, however, Kester et al. 2017). The overall calibration uncertainty, assumed to amount to $\sim 10 \%$, is irrelevant here because we need only the line-to-continuum ratio.

\section{Appendix B: Assessment of asymmetric errors}

Column densities directly derived from substantial optical depths, which in turn are determined from the line-to-continuum ratio in absorption spectra, are fraught with asymmetric errors. The accuracy of the line-to-continuum ratio (hereafter referred to as $r$ ) is limited by radiometric, Gaussian baseline noise, and, sometimes, the uncertainty of the continuum level. This leads to uncertainties in the fitted line-to-continuum ratio, of which the non-linear transformation to column densities lead to asymmetric errors. The analysis applied in this work follows Barlow (2003): in his model (1) the errors in the derived quantity are estimated by straight lines leaving from the central value, with slopes of $\sigma^{+}$and $\sigma^{-}$when the normal distribution of $r$ is transformed to a unit Gaussian. With the given opacity mean $\tau_{\mu}=\langle\tau\rangle$, its variance $V=\left\langle\tau^{2}\right\rangle-\tau_{\mu}^{2}$ and the unnormalized skewness, $\gamma=$ $\left\langle\tau^{3}\right\rangle-3 \tau_{\mu}\left\langle\tau^{2}\right\rangle+2 \tau_{\mu}^{3}$, at hand, this leads to a system of three coupled, nonlinear equations for the median $\tau_{\mathrm{m}}$ and the asymmetric errors $\sigma_{\tau,+}$ and $\sigma_{\tau,-}$, namely,

$$
\begin{aligned}
\tau_{\mathrm{m}}= & \tau_{\mu}-\frac{1}{\sqrt{2 \pi}}\left(\sigma_{\tau,+}-\sigma_{\tau,-}\right), \\
f_{1}= & \frac{1}{4}\left(\left(\sigma_{\tau,+}+\sigma_{\tau,-}\right)^{2}+\left(\sigma_{\tau,+}-\sigma_{\tau,-}\right)^{2}\left(1-\frac{2}{\pi}\right)\right)-V, \\
f_{2}= & 2\left(\sigma_{\tau,+}^{3}-\sigma_{\tau,-}^{3}\right)-\frac{3}{2}\left(\sigma_{\tau,+}-\sigma_{\tau,-}\right)\left(\sigma_{\tau,+}^{2}+\sigma_{\tau,-}^{2}\right) \\
& +\frac{1}{\pi}\left(\sigma_{\tau,+}-\sigma_{\tau,-}\right)^{3}-\gamma \sqrt{2 \pi}
\end{aligned}
$$

Model (2) of Barlow (2003) considers a parabolic fit through the three points (i.e., the central value and $\pm \sigma$ ), rather than the piecewise defined straight lines of model (1). The above system of equations for $\sigma_{\tau, \pm}$ and $\tau_{\mathrm{m}}$ then reads

$$
\begin{aligned}
\tau_{\mathrm{m}} & =\tau_{\mu}-\frac{1}{2}\left(\sigma_{\tau,+}-\sigma_{\tau,-}\right), \\
f_{1} & =\frac{1}{2}\left(\sigma_{\tau,+}+\sigma_{\tau,-}\right)^{2}+\frac{1}{2}\left(\sigma_{\tau,+}-\sigma_{\tau,-}\right)^{2}-V, \\
f_{2} & =\frac{3}{4}\left(\sigma_{\tau,+}+\sigma_{\tau,-}\right)\left(\sigma_{\tau,+}-\sigma_{\tau,-}\right)+\left(\sigma_{\tau,+}-\sigma_{\tau,-}\right)^{3}-\gamma .
\end{aligned}
$$

The solution of Eq. (B.1) or (B.2) is determined (iteratively or graphically) by the crossings of the $f_{1}\left(\sigma_{\tau,+}, \sigma_{\tau,-}\right)=0$ and the $f_{2}\left(\sigma_{\tau,+}, \sigma_{\tau,-}\right)=0$ contours. The resulting $\left(\sigma_{\tau,+}, \sigma_{\tau,-}\right)$ pair is subsequently used to determine $\tau_{\mathrm{m}}$ from the first equation of the set B.1 or B.2 (for model (1) and model (2), respectively).

A demonstration of this approach is shown in Fig. B.1, for a two-component opacity profile (a saturated absorption of $\tau_{0}=8$, and an unsaturated yet substantial one of $\tau_{0}=2$, located in the wing of the stronger absorption). We note that in both cases the median value determined with the above models is within $1 \sigma$ limits from the original values, with the stronger deviation for the saturated case. We also note that the line center opacity in the saturated component could be determined thanks to the curvature of the line profile in its unsaturated line wing opposite to 

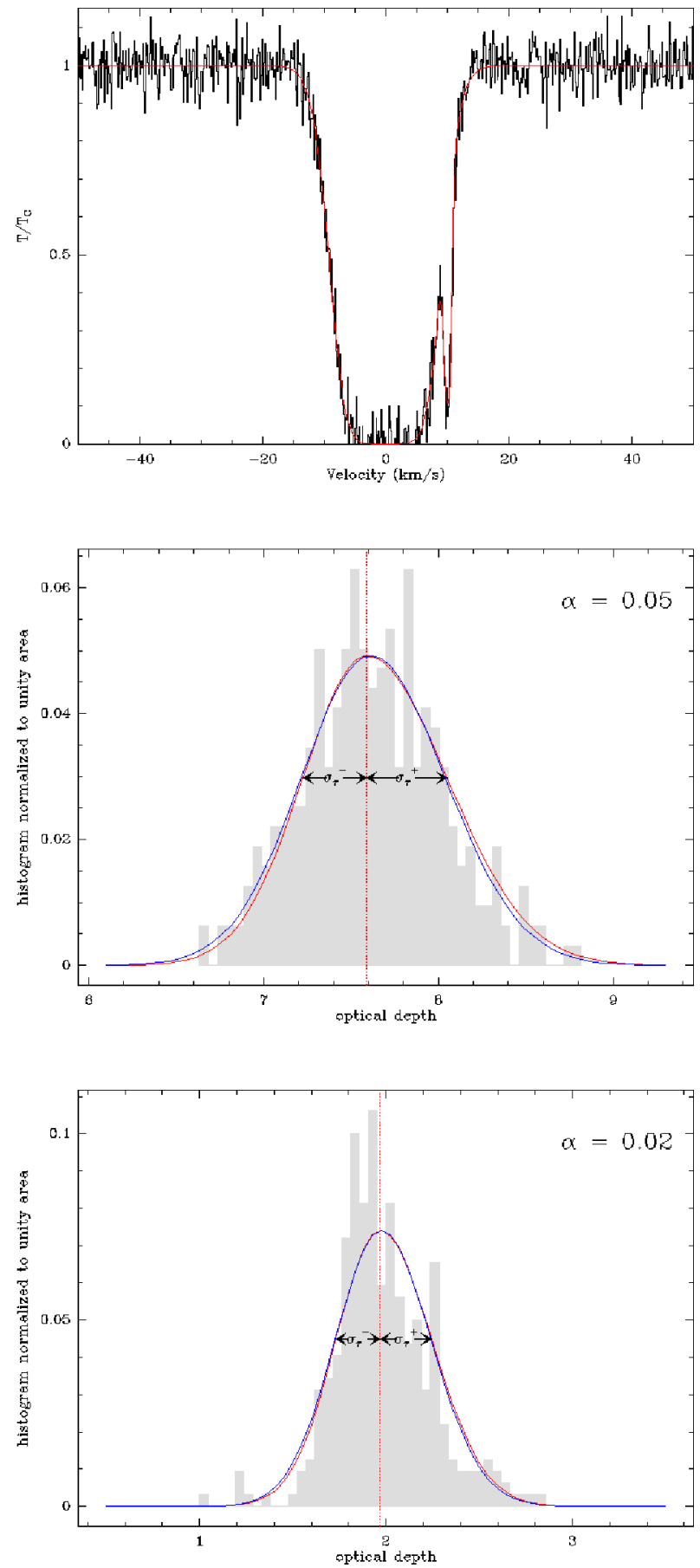

Fig. B.1. Demonstration of Bayesian error estimation according to Barlow (2003). Top panel: test profile. The signal-to-noise ratio of the continuum is 20. Center and bottom panels: histograms of the optical depth distribution, for the saturated absorption (center panel) and the narrower, unsaturated component (bottom panel). The dimidated Gaussians from models (1) and (2) are shown in red and blue, respectively, along with the asymmetric errors (arrows) and the asymmetry parameters $\alpha$.

the wing that contains the unsaturated component. As expected, the asymmetry parameter $\alpha=\left(\sigma_{\tau,+}-\sigma_{\tau,-}\right) / 2$ is larger for the saturated absorption component. One can also see that the difference between both models is almost unnoticeable and irrelevant. Without loss of generality, the error analysis applied in this paper relies on model (1).

\section{Appendix C: Comparison of column densities derived from the $\lambda 560 \mu \mathrm{m}$ and $\lambda 149 \mu \mathrm{m}$ transitions}

A comparison of $\mathrm{CH}$ column densities derived from the $\lambda 149 \mu \mathrm{m}$ and the $\lambda 560 \mu \mathrm{m}$ lines may be instructive here. The weighted linear regression is shown in Fig. C.1. Assuming a 5\% error in the continuum level, the column densities derived from the $\lambda 149 \mu \mathrm{m}$ line are systematically $(-0.40 \pm 0.05) \times 10^{13} \mathrm{~cm}^{-2}$ below those derived from the $\lambda 560 \mu \mathrm{m}$ line, with a slope of $(0.93 \pm 0.03) \times 10^{13} \mathrm{~cm}^{-2}(\lambda 149 \mu \mathrm{m}$ vs. $\lambda 560 \mu \mathrm{m})$. It seems fair to say that the explanation for this discrepancy is either one of the following: (1) as stated in the introduction, the determination of column densities derived from the $\lambda 560 \mu \mathrm{m}$ spectra needs to make an ad-hoc assumption about the emission profile. Due to the mix of emission and absorption around the hot core, the emission component is difficult to define, but subsequently needed to disentangle the sightline absorption from the emission component (which folds into the absorption by the spiral arms owing to the relatively large hyperfine splitting). (2) Any inconsistency in the calibration of the continuum level (e.g., of the contribution from the image band), with respect to that of the spectral line, will lead to a corresponding error in the opacity determination (cf. the discussion in Appendix A). The common assumption that all $\mathrm{CH}$ molecules in the diffuse gas are in the ground state is unlikely to be invalid here, as shown in Table D.1. Without a deeper analysis, it will therefore be difficult to identify the origin of the discrepancy, and it seems both suggestions made above are at work.

\section{Appendix D: Non-LTE effects in the hyper-fine split $\mathrm{CH}$ ground state}

Here we provide a short demonstration of NLTE effects in the ground state level populations of $\mathrm{CH}$ under conditions typical for diffuse clouds. Table D.1 lists the departure coefficients for two models, one corresponding to a diffuse cloud with low molecular content, the other one to a translucent cloud with a substantial fraction of molecules; the calculations have been performed with the MOLPOP-CEP code (Elitzur \& Asensio Ramos 2006), assuming a simple slab model. All rotational $\mathrm{CH}$ levels up to $E_{\mathrm{u}}=1120 \mathrm{~K}$ were considered. This implies radiative pumping, externally by the interstellar radiation field (ISRF), as given by Mathis et al. (1983) and Draine (1978), and by the cosmic microwave background, and internally by dust heated by the ISRF (resulting dust temperatures are from Voshchinnikov et al. 1999). The quantities defining the clouds are from Snow \& McCall (2006). The escape probability is from Capriotti (1965). Alternatively, the exact CEP solution was used, the relative differences in the level populations amount to at most $1-3 \%$. For both techniques we assume a static cloud with a turbulent Doppler width of $2 \mathrm{~km} \mathrm{~s}^{-1}\left(3.3 \mathrm{~km} \mathrm{~s}^{-1} \mathrm{FWHM}\right)$. This choice is at the lower end of the linewidth-size relation for diffuse gas on spatial scales from 10 to $100 \mathrm{pc}$ (Falgarone et al. 2009) and close to the median width resulting from fits to $\mathrm{OH}^{2} \Pi_{3 / 2}, J=5 / 2 \rightarrow 3 / 2$ absorption line systems, observed on nine sightlines through the first and fourth quadrant of the Galaxy (Wiesemeyer et al. 2016). Collision rates are from Marinakis et al. (2015, scaled from collisions with $\mathrm{He}$ to those with $\mathrm{H}$ and $\mathrm{H}_{2}$ ). The departure from LTE is model-dependent and varies within an order of magnitude. 

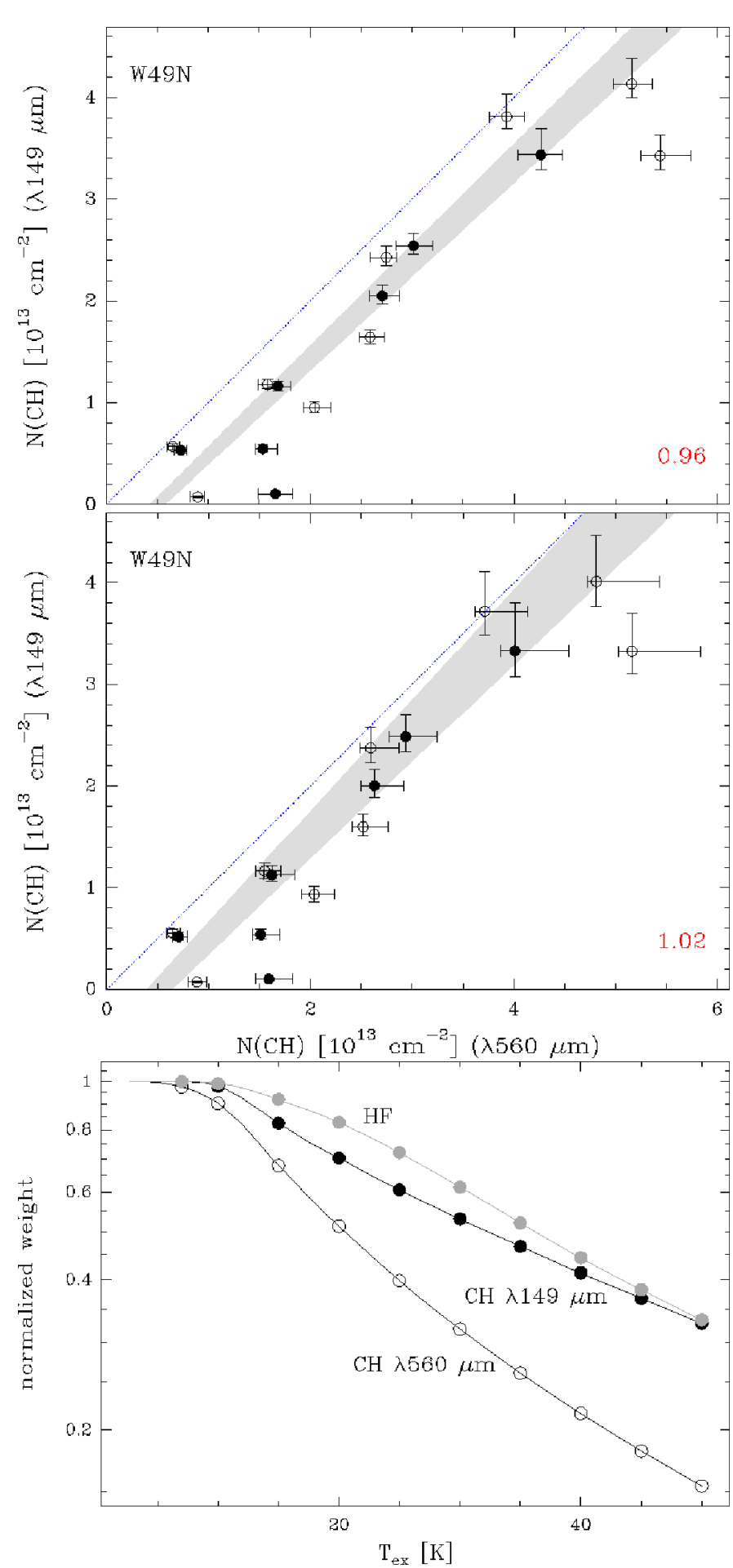

Fig. C.1. Comparison of column densities derived from the $\lambda 149 \mu \mathrm{m}$ and $\lambda 560 \mu \mathrm{m}$ lines, assuming a 5\% error in the continuum level (top panel) and a 10\% error (center panel). The gray-shaded area covers all data points within the $1 \sigma$ limits of the weighted linear regression (derived slopes in the bottom right corners). The column densities refer to $3 \mathrm{~km} \mathrm{~s}^{-1}$ wide velocity intervals in the spectral distribution of $N_{\mathrm{v}}$ (cf. Fig. 2). Only velocities above $30 \mathrm{~km} \mathrm{~s}^{-1}$ were considered. The dotted blue line indicates the bisector for identical column densities derived from both lines. Bottom panel: normalized statistical weights to convert the total column densities to opacities.
Table D.1. Conditions in diffuse cloud models and coefficients for departure from LTE of the population in the CH $N=1, J=1 / 2 \mathrm{HFS}$.

\begin{tabular}{|c|c|c|}
\hline & $\begin{array}{c}\text { Model } 1 \\
\text { diffuse molecular }\end{array}$ & $\begin{array}{c}\text { Model } 2 \\
\text { translucent }\end{array}$ \\
\hline$\chi[$ Habing $]$ & 1.7 & 1.7 \\
\hline$A_{\mathrm{V}}$ & 0.2 & 1 \\
\hline$n_{\mathrm{H}}\left[\mathrm{cm}^{-3}\right]$ & 100 & 1000 \\
\hline$f_{\mathrm{H}_{2}}^{\mathrm{n}}$ & 0.1 & 0.5 \\
\hline$T_{\text {gas }}[\mathrm{K}]$ & 100 & 15 \\
\hline$T_{\text {dust }}[\mathrm{K}]$ & 16 & 12 \\
\hline$\Delta v_{\text {FWHM }}\left[\mathrm{km} \mathrm{s}^{-1}\right]$ & 3.3 & 3.3 \\
\hline$N(\mathrm{CH})\left[10^{13} \mathrm{~cm}^{-2}\right]$ & 1.75 & 1.58 \\
\hline \multicolumn{3}{|c|}{ LTE departure coefficients } \\
\hline$F=0+$ & 3.702 & 0.5480 \\
\hline$F=1+$ & 3.721 & 0.5492 \\
\hline$F=0-$ & 6.875 & 2.178 \\
\hline$F=1-$ & 6.948 & 2.199 \\
\hline \multicolumn{3}{|c|}{ Fractional population (per sub-level) in ground state } \\
\hline$F=0+$ & 0.0870 & 0.0504 \\
\hline$F=1+$ & 0.0875 & 0.0505 \\
\hline$F=0-$ & 0.1613 & 0.1981 \\
\hline$F=1-$ & 0.1630 & 0.2000 \\
\hline \multicolumn{3}{|c|}{ Fractional population in excited states [ppm] } \\
\hline & 100 & 200 \\
\hline
\end{tabular}

Notes. Departure coefficients are defined as the fractional level population with respect to LTE. The FUV field is parametrized in units of the Habing field (Draine field = 1.7 Habing, Draine 1978). 